\title{
Augmented Lagrangian simulations study of yield-stress fluid flows in expansion-contraction and comparisons with physical experiments $^{1}$
}

\author{
November 22, 2016
}

Arthur Marly ${ }^{2,3}$, Paul Vigneaux $2,3, *$

\begin{abstract}
We present numerical simulations of viscoplastic flows in expansion-contraction geometry and compare them with physical experiments of [Chevalier et al. Europhys. Lett. 102, 48002 (2013)] and [Luu et al. Phys. Rev. E 91, 013013 (2015)]. Numerical resolution is done with Augmented Lagrangian (following the Glowinski and coworkers' approach) and Finite-Differences (for the space discretization) methods. We show that good agreement is obtained between the numerical results and the physical experiments. In particular, we retrieve the slip line effect of Luu et al. and give numerical evidence of non-monotone shear effect in the boundary layer between the two unyielded regions in the cavity region. We also give some more detailed measures of the size of the plug and dead zones.
\end{abstract}

Keywords: Viscoplastic flows, Bingham fluid, expansion-contraction geometry, yielded boundary layer, Augmented Lagrangian methods, Finite-Differences, Parallel multifrontal solver

\section{Introduction}

In the present article, we study the ability of Augmented Lagrangian methods to simulate two dimensional flows of viscoplastic materials in rectangular expansion-contraction geometries. We are specifically interested in the numerical simulation of recent physical experiments of Chevalier et al. [6] and Luu et al. [19]. We provide a detailed analysis of the velocity profiles and unyielded zones.

Even if the fluids used in the above experiments are described by a Herschel-Bulkley law, we restrict ourselves to a Bingham constitutive law since, as mentioned in the PhD thesis of Chevalier, it still allows to have good insight of these viscoplastic flows. This will be confirmed in the present article. Moreover for such experiments, flows are studied when reaching a stationary state.

\footnotetext{
${ }^{1}$ Authors' version on HAL. Published in Journal of Non-Newtonian Fluid Mechanics 239 (2017) 35 52. DOI, direct link

${ }^{2}$ Univ Lyon, ENS de Lyon, UMPA, CNRS UMR 5669. F-69364 Lyon, France

${ }^{3}$ INRIA, NUMED Team. F-69364 Lyon, France. * Corresp. author: Paul.Vigneaux@math.cnrs.fr
} 
Precisely, we thus want to solve the following 2D stationary, so called, Stokes-Bingham problem:

$$
\left\{\begin{aligned}
-\nabla \cdot \tau+\nabla p & =0 \\
\nabla \cdot \mathbf{u} & =0
\end{aligned}\right.
$$

where $\tau$ is given by the Bingham constitutive law:

$$
\left\{\begin{array}{c}
\tau=2 \eta D(\mathbf{u})+\tau_{y} \frac{D(\mathbf{u})}{|D(\mathbf{u})|} \Leftrightarrow D(\mathbf{u}) \neq 0 \\
|\tau| \leqslant \tau_{y} \Leftrightarrow D(\mathbf{u})=0 .
\end{array}\right.
$$

The viscosity of the viscoplastic material is denoted by $\eta$ and the yield stress by $\tau_{y}$. We denote by $D(\mathbf{u})$ the rate of deformation tensor: $D(\mathbf{u})=\left(\nabla \mathbf{u}+\nabla \mathbf{u}^{t}\right) / 2, \mathbf{u}=(u, v)$ and by $p$ the pressure. We also use the following convention: for a tensor $\tau$, we use the norm $|\tau|^{2}=\frac{1}{2} \sum_{i j} \tau_{i j}^{2}$. The stress of the material is below the $\tau_{y}$ threshold when the material is rigid $(D(\mathbf{u})=0$, also called the unyielded state). On the contrary, the material is deformed with a linear law for any stress above $\tau_{y}$. This kind of viscoplastic formalism originated independently from the works of Schwedoff [31] and Bingham [3], and was then extended to the 3D tensorial form by Prager [17].

The numerical simulation of Bingham flows generated a wide variety of methods to deal with the main difficulty of such problem, namely the fact that the constitutive law is multivalued when the stress is below $\tau_{y}$. For an extensive review, we refer to the book of Glowinski and Wachs [15]. In brief, one can distinguish two families of approaches: on the one hand, regularization approaches which make the Bingham law univalued and allow to solve (1)-(2) in the strong form, using classical methods as for the incompressible Stokes equation. Of note, even if sometimes very interesting from the theoretical PDE point of view, regularization approaches may lead from the computational viewpoint to wrong computation of the yield surfaces associated to the exact Bingham model, see [12] for a review: an example of such problem is given by Burgos et al. [4] where a simulated yield surface has the inverse convexity of the true expected analytic yield surface.

On the other hand, one can use variational approaches where (1)-(2) is reformulated as a variational inequality which allows to solve more precisely for the rigid zones. They can be traced to the works of Il'iushin [18], Prager [24], Mosolov and Miasnikov [20] and Duvaut and Lions [10]. Efficient numerical techniques where designed following the works of Glowinski, Lions and Trémolières [10] and coworkers, including the so called Augmented Lagrangian (AL) methods which are used in the present paper and will be described in the following section. We refer again to [15] and references therein for numerous applications of AL methods in the simulation of viscoplastic flows.

Of course, since it is widely used in practical applications, the expansion-contraction geometry has been studied in many previous works. Let us mention the work of de Souza et al. [7] which seems to be the first work close to the present study: they present experiments with Carbopol and compare with numerical solutions obtained with a regularization method. In addition, similar configurations are simulated in [28] and [29] using an AL method on unstructured meshes. Their code is implemented with the excellent Rheolef library [30] of Saramito and coworkers (see e.g. [26] for the flow around a cylinder). An impressive range of Bingham numbers, aspect ratios of the geometry and shapes of the cavity (rectangular, sinusoidal wave, triangular, semi-fractal) are presented. But they did not describe in depth the velocity profiles in conjunction with the plug zone, along the lines of the physical experiments of Coussot's and Chambon's groups [6, 19].

The characteristics of the present paper are the following. 
- As said previously we use an AL approach and we adopt a finite-difference approach (on Cartesian meshes) for the discretization in space. This is in the spirit of Wachs and co-workers (see [32] or, for a longer description, [15]), as well as E. Muravleva, A. Muravleva, Olshanskii and coworkers (see e.g. [21], [22]) but our implementation differs on the resolution of the induced generalized Stokes problem which is here also tackled with another AL approach (to fulfill the incompressibility condition). See section 2.2. In addition, we make a finely tuned use of parallel linear system solvers which helps in using very fine (isotropic) Cartesian meshes, not that often published in the simulation of viscoplastic flows considered here.

- Code results are scrutinized in terms of accuracy of the localization of the plastic zone and computational times, given the fact that we impose a really small residue $\left(\sim 10^{-12}\right)$ in the AL loop : such information are rarely given in the associated literature and can serve for future comparisons.

- As a validation/application of the code, we retrieve in section 3 the results of the frustrated regime studied in [6] and additionally show the evolution of the yielded boundary layer width as a function of the Bingham number. We also retrieve the existence of a so-called slip line and the Poiseuille-like behaviour above this slip line shown in [19] (see section 4). Of note, we also give the horizontal length of the dead zone at the corner of the cavity as a function of the Bingham number (section 2.3).

\section{Expansion-contraction channel simulations}

\subsection{Description of the problem}

The geometry and notations of the expansion-contraction problem are illustrated in figure 1 , where only the upper half is shown. In the following, we will use either (1)-(2) or their dimensionless form (by denoting dimensionless variables with a tilde symbol) which reads:

$$
\left\{\begin{aligned}
-\tilde{\nabla} \cdot \tilde{\tau}+\tilde{\nabla} \tilde{p} & =0 \\
\tilde{\nabla} \cdot \tilde{\mathbf{u}} & =0
\end{aligned}\right.
$$

with

$$
\left\{\begin{array}{c}
\tilde{\tau}=2 \tilde{D}(\tilde{\mathbf{u}})+B \frac{\tilde{D}(\tilde{\mathbf{u}})}{|\tilde{D}(\tilde{\mathbf{u}})|} \Leftrightarrow \tilde{D}(\tilde{\mathbf{u}}) \neq 0 \\
|\tilde{\tau}| \leqslant B \Leftrightarrow \tilde{D}(\tilde{\mathbf{u}})=0 .
\end{array}\right.
$$

In this dimensionless Stokes-Bingham model, there is a unique dimensionless number $B=\frac{\tau_{y} D}{\eta \bar{U}}$, called the Bingham number, where $D$ is the small channel half-width (see figure 1 ) and $\bar{U}$ is the mean flow velocity in the $x$-direction at the entrance (see (5)). Indeed, the dimensionless model is obtained from (1)-(2) by scaling the lengths with $D$, the velocities with $\bar{U}$ and stresses with $\frac{\eta \bar{U}}{D}$. In dimensional variables, we have

$$
\bar{U}=\frac{1}{D} \int_{0}^{D} u(0, y) \mathrm{d} y
$$

We consider the two following aspect ratios:

$$
h=\frac{D+H}{D} \quad \text { and } \quad \delta=\frac{D}{L} .
$$



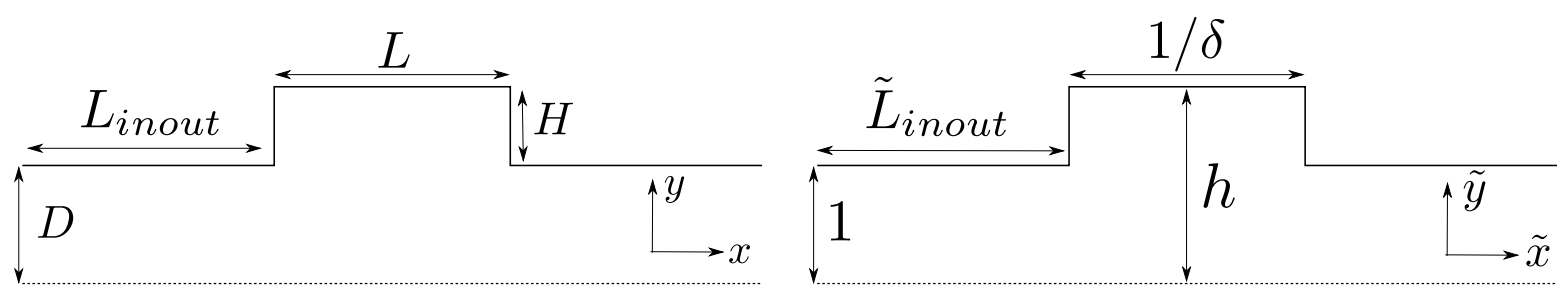

Figure 1: Geometry of the expansion-contraction and notations for the dimensional form of the model (left, cf. eqs. (1)-(2)) and the dimensionless one (right, cf. eqs. (3)-(4)). Only the upper half is represented thanks to the symmetry w.r.t. the $x$-axis.

In the inlet and outlet, we set the flow equal to the Poiseuille flow (with a unit net flux) in the infinitely long channel. At the lateral wall, the velocity is set equal to 0. See Appendix A.2 for details. Of note, in section 3, we will present the results in the dimensionless form, but we will use the dimensional form in section 4 to compare more easily with the results of $[19]$.

To sum up, in dimensionless variables, the free parameters are $h, \delta$ and the Bingham number $B$.

\subsection{Salient features of the numerical results}

As said in the introduction, we implemented an Augmented Lagrangian method as in the seminal work of Glowinski and coworkers $[14,8]$. The discretization in space is done with finite-differences on rectangular grids. As such, present work is complementary to $[28,29]$ since it allows to compare the results between structured and non-structured grids discretizations. For completeness and reproducibility of the paper, we give in Appendix A the algorithms we implemented with Fortran 90 and MPI.

The first key point is that the simulations presented in the paper are much more converged in terms of the AL iterations than many of the associated simulations previously published. For instance, instead of enforcing a convergence of $10^{-6}$ for the Bingham AL loop's convergence criterion, we used $6 \cdot 10^{-12}$ (and also validated the code up to machine precision $10^{-15}$ ). The second important point is that the linear systems which need to be solved are handled by the MUMPS library $[1,2]$. This massively parallel solver allows us to use very fine meshes up to $7.8 \cdot 10^{6}$ points and to obtain computational times shorter than 2 days on 16 cores.

Figure 2 shows typical computed velocity, pressure and $|\tilde{d}|$ (which approximates $|\tilde{D}(\tilde{\mathbf{u}})|$ as shown in Algorithm 1, cf. Appendix A.1) fields, for $\delta=0.5, h=2$ and $B=5$. We directly remark that velocity, pressure and deformation are symmetric with respect to both middle axis in the $\tilde{x}$ and $\tilde{y}$ directions (and it is the same for the stress tensors). Hence, often in the sequel, we only show the upper-left quarter of the domain. Further, as often done in the literature, we cover the plastic zones in the stress fields with a black patch since there's no consistent notion of pressure or stress in the rigid zone, for the Bingham model (1)-(2).

Let us detail how we localize the plastic zones in the following sections. In figure 2 (bottom, right), instead of showing, as in the pressure field on its left, just one level of $\tilde{D}(\tilde{\mathbf{u}})$ (or $\tilde{d}$, which is virtually equivalent, see next paragraph), we show the whole deformation field in log scale since it allows the reader to have a precise idea on how the 

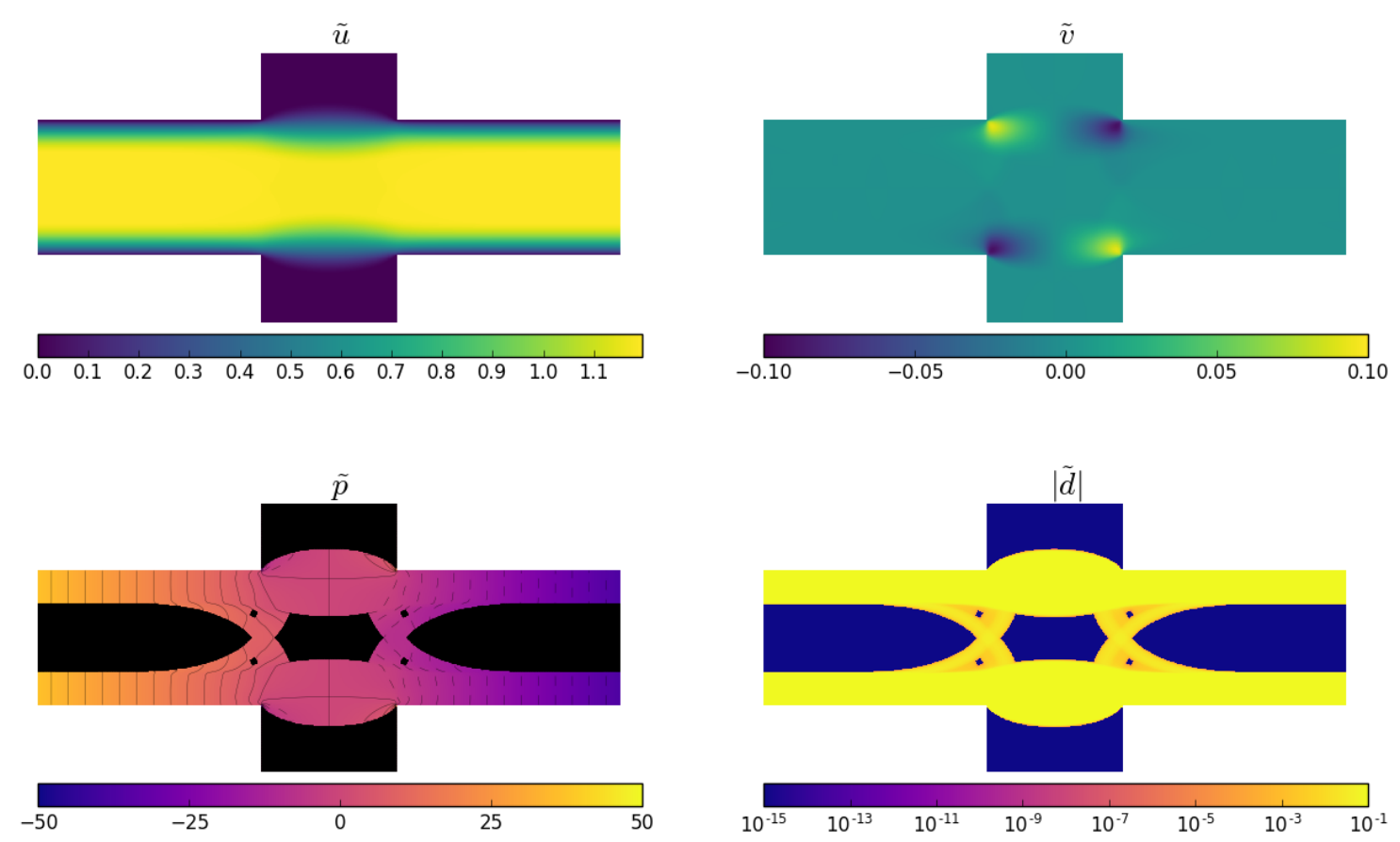

Figure 2: Solution computed for $\delta=0.5, h=2$ and $B=5$, dimensionless variables.

numerical method is performing. Of note, this presentation is not quite often done in the literature: to our knowledge [25] is one of the very few published papers showing the complete Lagrange multiplier, but, still, not in log scale, leading to a less clearer view (in particular in the pseudo-plug region, see below). Two main features appear in this case:

- First, we observe a huge slope of the deformation and of the multiplier in the region of the yield surface; this is visible with a very fast transition (almost a single line) in log scale between $10^{-15}$ and $10^{-1}$ at the edge of the dead zone in the cavity, at the horizontal edges of the central disconnected plug and in most of the entry/exit channels. Here, the plastic zone is very clearly seen. This is a sign of a very good quality of the convergence of Algorithm 1.

- Second, it is also interesting to note that a pseudo-plug zone [25] can be seen (by looking at the values between $\left.\left[10^{-5} ; 10^{-3}\right]\right)$ surrounding the disconnected plug located in the center of the domain (see also figure 3 for a zoom). Of note, inside this pseudo-plug zone, one can also see four little "square" plugs which are also exhibited in $[29,27]$. These four plugs are confirmed under mesh refinement (see below).

Let us have a closer look at the plastic zone indicators. The figure 3 shows $|\tilde{D}(\tilde{\mathbf{u}})|, \tilde{d}$ and their difference, all in the $\log$ scale. We see that both $|\tilde{D}(\tilde{\mathbf{u}})|$ and $\tilde{d}$ are good indicators of the plastic zones but $\tilde{d}$ is slightly better. This is clearly understood by the structure of Algorithm 1 for which the plastic threshold is directly used in the computation of $\tilde{d}$ (which is local), whereas $|\tilde{D}(\tilde{\mathbf{u}})|$ is obtained by discrete differentiation of $\tilde{\mathbf{u}}$. Quantitatively, it appears that taking the zone $\left\{|\tilde{d}|=10^{-15}\right\}$ (the machine precision for 0 ) or an upper level belonging to $\left.\left[10^{-10} ; 10^{-6}\right]\right)$ to delimit the plastic zone leads to the same results. In the rest of the paper, we will thus present the $|\tilde{d}|$ fields (instead of $|\tilde{D}(\tilde{\mathbf{u}})|$ ) of our various simulations to localize the plastic zones.

Figure 4 shows the evolution of the plastic zone (defined here as the level $|\tilde{d}|=10^{-10}$ ) when the mesh is refined. One can observe that these lines are very close to one another. 


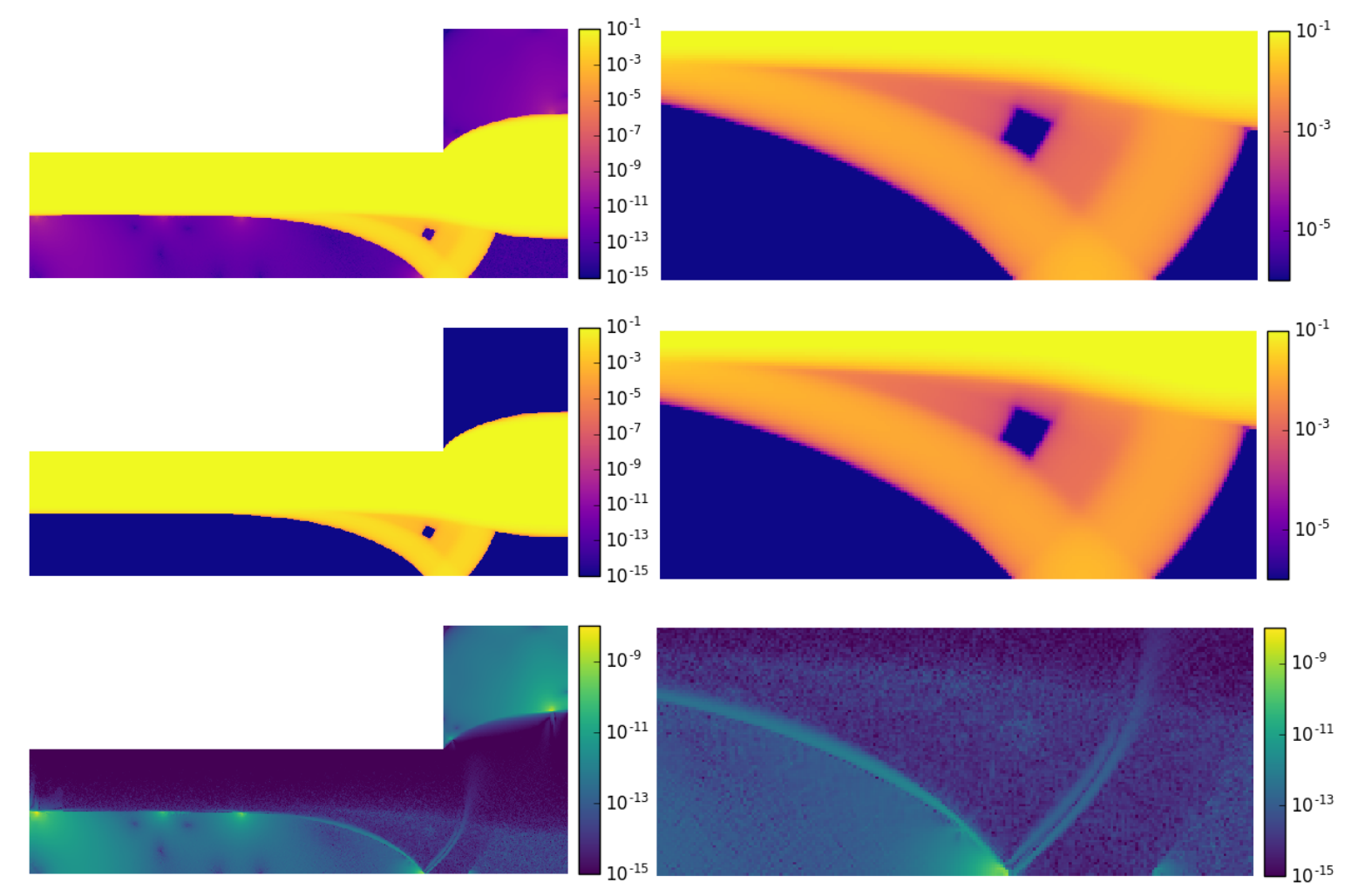

Figure 3: Localization of the plastic zone with log scale (zoom of fig. 2). $\delta=0.5, h=2$ and $B=5$. From top to bottom: $|\tilde{D}(\tilde{\mathbf{u}})|, \tilde{d}$ and the residue $|\tilde{D}(\tilde{\mathbf{u}})-\tilde{d}|$. Left: the whole quarter domain. Right: zoom of Left on the pseudo-plug zone, remark that the colormap is refined between $\left[10^{-6} ; 10^{-1}\right]$.

Moreover, the volume of the plug zone seems to increase monotonically and converges to a limit plug. This convergence is another sign of the well-posedness of the algorithm and discretization. The fact that one needs to zoom to point out these minor differences invites us to use the less refined mesh. In the rest of the paper, otherwise stated, we set the length of a cell such that there are 600 points in a section of cavity. This condition ensures a dramatic reduction of CPU time without a significant loss of accuracy.

Of note, the meshes used in the figure 4 have from 1.25 to $7.8 \cdot 10^{6}$ cells and the CPU times were between $5 \mathrm{~h} 30$ and $32 \mathrm{~h} 30$. Since the literature is very scarce in terms of CPU times information for such simulations, the interested reader can find in Appendix A.3 the computation times of a wide range of simulations done in this paper, which can be used for future comparisons.

\subsection{Different plastic zones}

Typical plastic domains are displayed in figures 5, 6, 7 and 8. In figure 5, we highlight the evolution of the plastic zones when $B$ increases. When $B$ is low, we observe a break in the plug zone whereas, when $B$ is high, the whole middle of the domain moves rigidly. In between (for instance $B=5$, in Magenta on figure 5), little patches of rigid material appear in the pseudo-plug zone, and they increase with $B$ to finally fusion within the continuous plug zone in the center of the channel.

Of course, the $B$ numbers where these patches appear, or where the whole length of the domain is plug, depends highly on the geometry and on the aspect ratios, cf. fig. 6, 7 


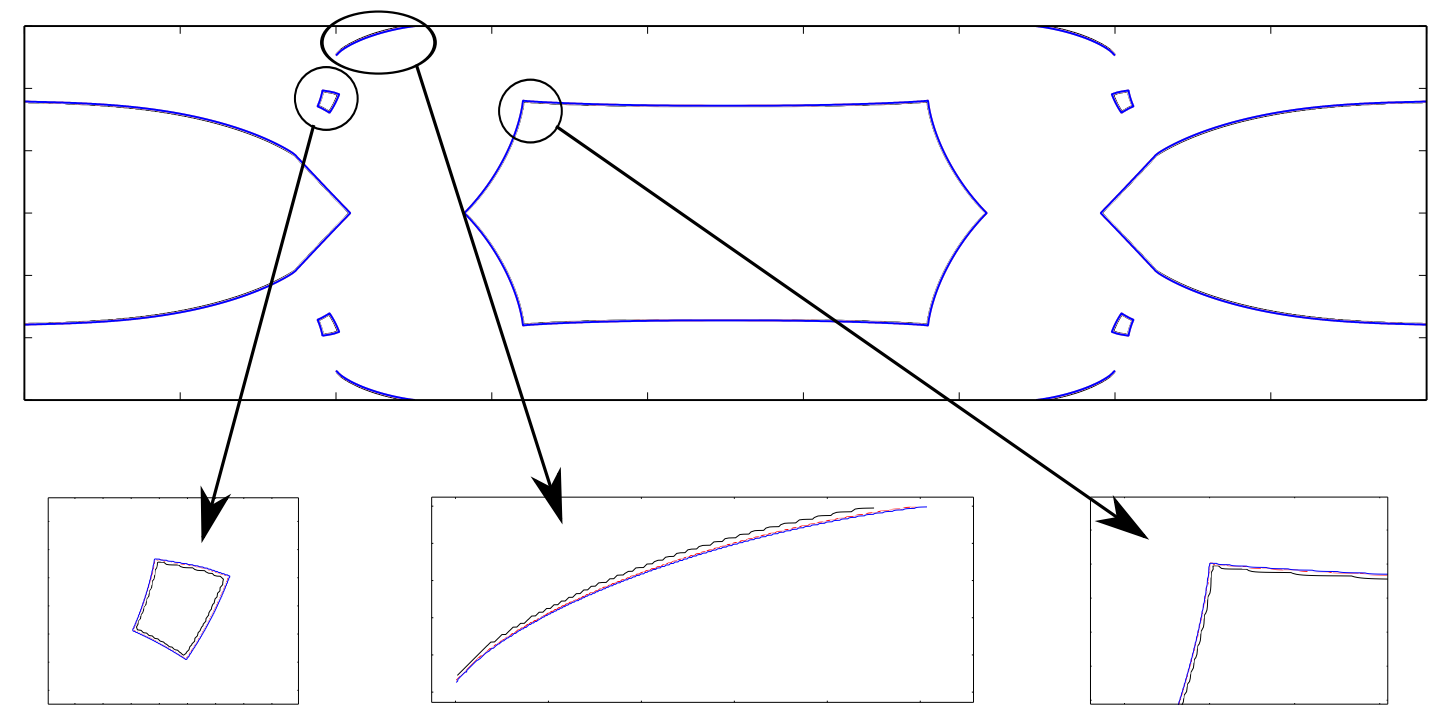

Figure 4: Mesh refinement study: $\delta=1 / 5, h=6 / 5$ and $B=20$. Contour $|\tilde{d}|=10^{-10}$ for $\Delta \tilde{x}=4 \cdot 10^{-3}$ (black), $\Delta \tilde{x}=2 \cdot 10^{-3}$ (red) and $\Delta \tilde{x}=1.6 \cdot 10^{-3}$ (blue).

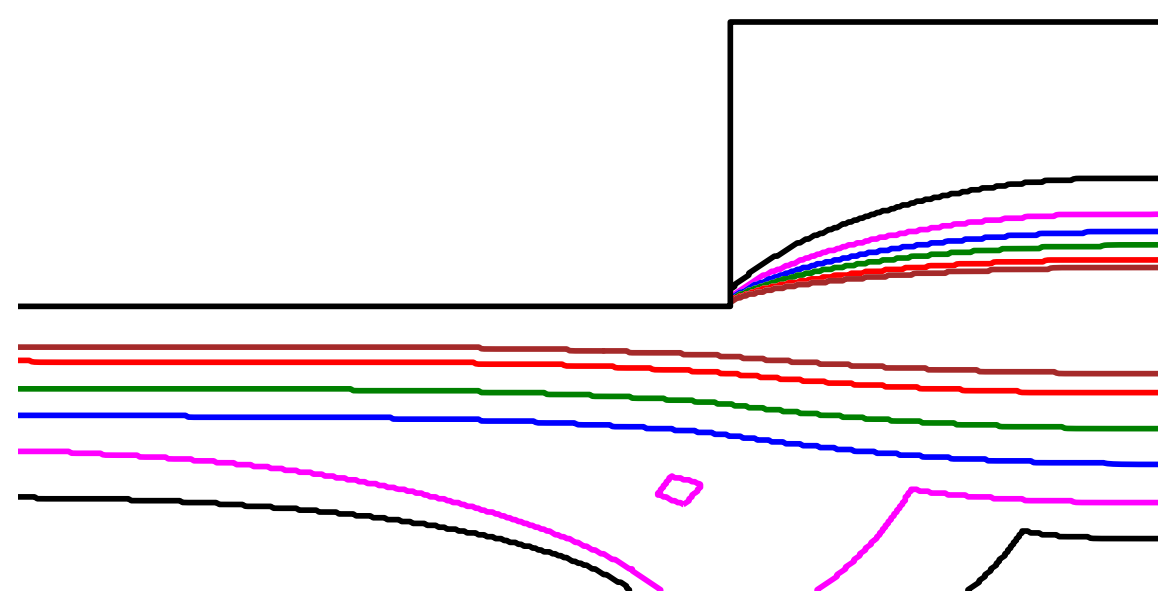

Figure 5: Different plastic zones. $\delta=0.5, h=1$ and various $B: 2$ (Black), 5 (Magenta), 10 (Blue), 20 (Green), 50 (Red) and 100 (Brown).

and 8. Present results compare well with Roustaei et al. [29], taking into account the fact that the geometry is here fully symmetric in $x$ and $y$, while [29] is only symmetric w.r.t. $y$.

In the case of "long" cavities as presented in Figure 8 and as in the configuration of [19], where the dead zone at the back of the cavity is disconnected, one can measure the horizontal length of the deadzone, denoted as $L_{d}$ (see Fig. 17, in section 4). Thanks to the simulations done for section 4 , we plotted $L_{d}$ as a function of the Bingham number. Figure 9 tends to show that this dependence follows a linear law in log-log coordinates. Finally, Appendix A.4 shows the same figure as 8 but with the full strain rate fields to better see the pseudo-plugs. 


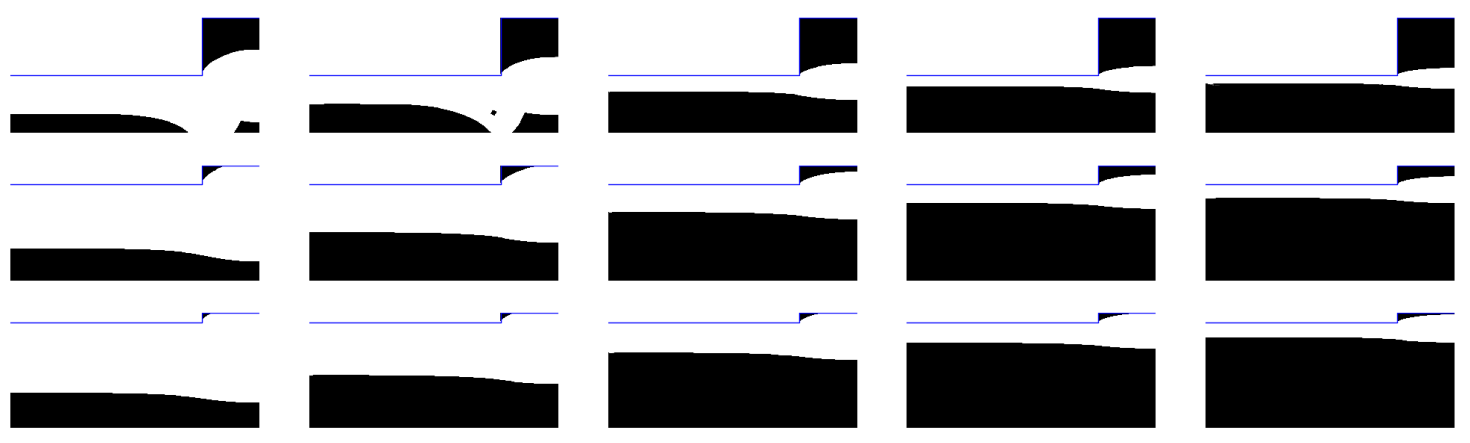

Figure 6: Different plastic zones: for various geometries and $B . h \delta=1$. From left to right, $B=2,5,20,50$ and 100 . From top to bottom, $h=2,6 / 5$ and $12 / 11$.

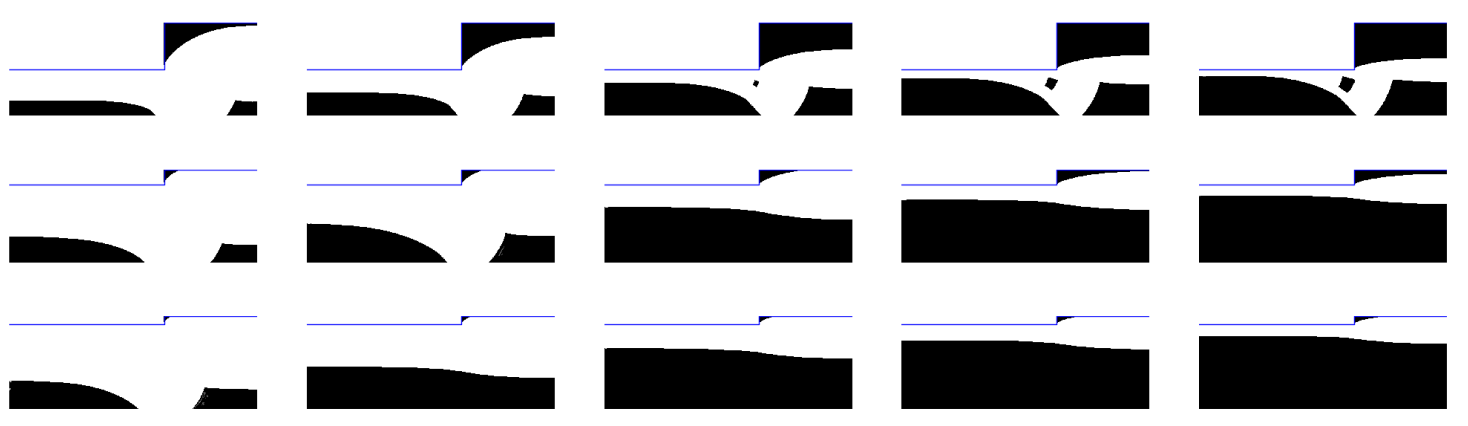

Figure 7: Different plastic zones: for various geometries and $B$. $h \delta=1 / 2$. From left to right, $B=2,5,20,50$ and 100 . From top to bottom, $h=2,6 / 5$ and $12 / 11$.

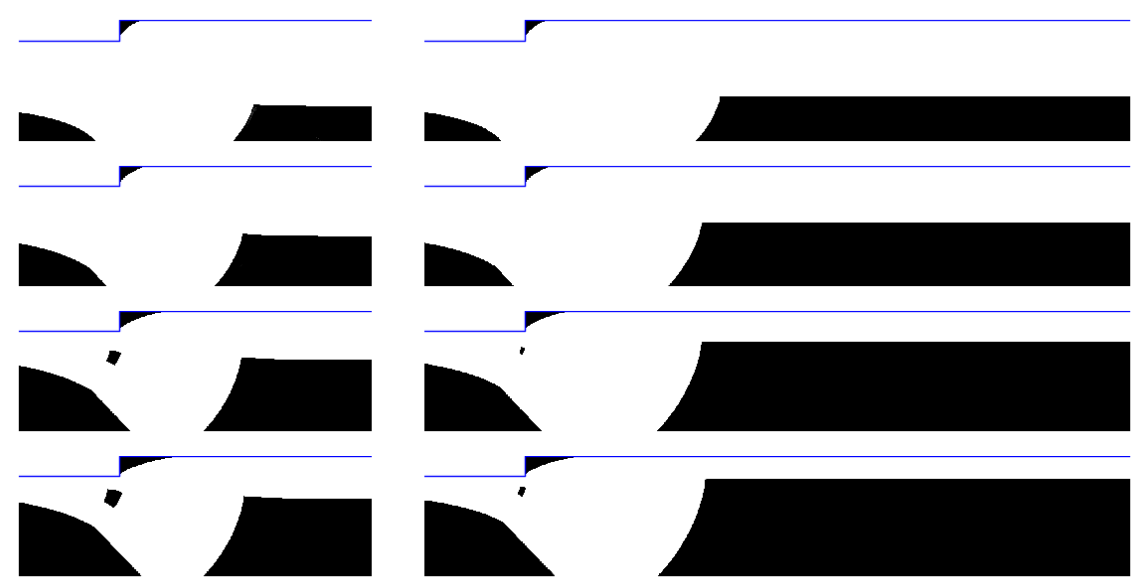

Figure 8: Different plastic zones for $h=6 / 5$ and $\delta=1 / 5$ (left) or $1 / 12$ (right). From top to bottom, $B=2,5,25$ and 50 . 


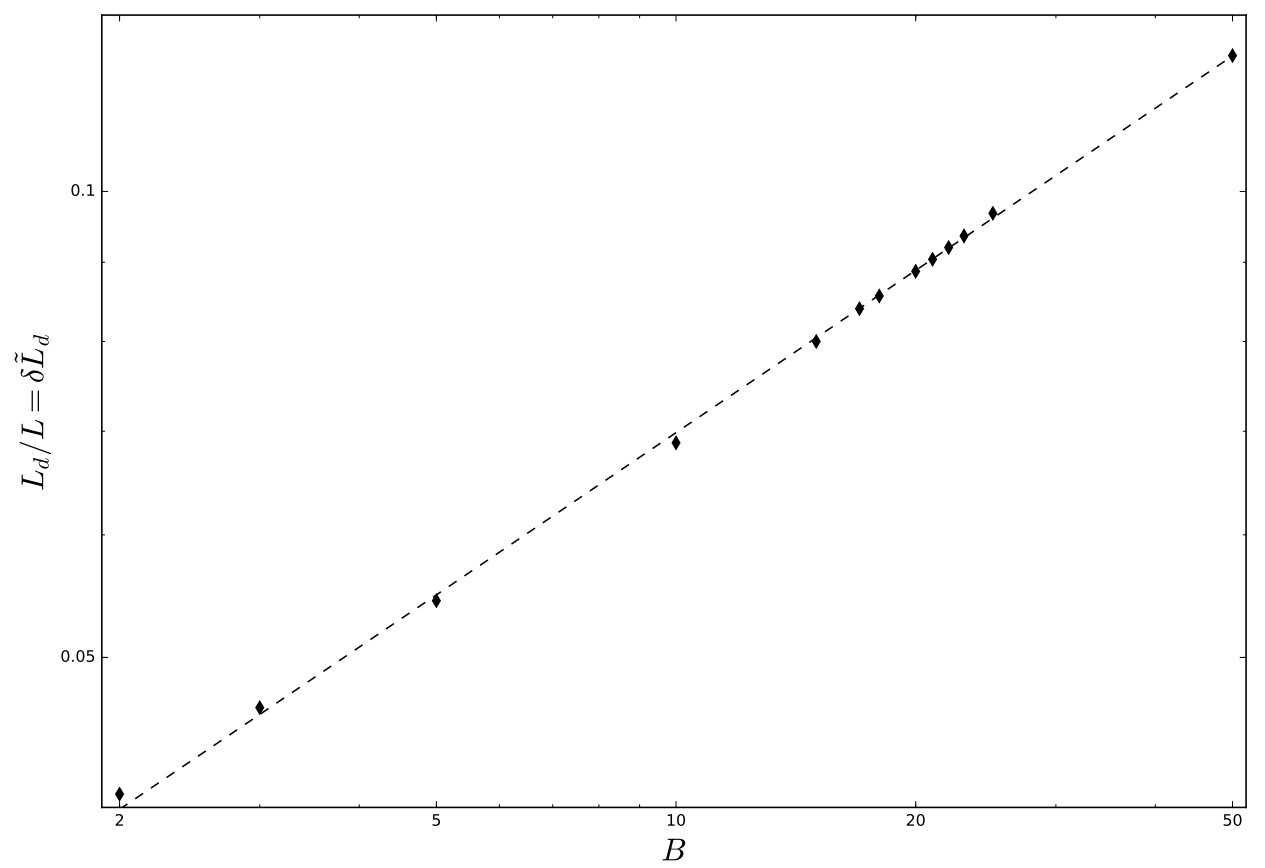

Figure 9: Length of the dead zone (scaled by $L$ ) as a function of $B$ in log-log scale (for $L=25$ and $\delta=1 / 5)$. A linear fit is done as a guide for the eye and the slope is 0.346 . 


\section{Experiments of Chevalier et al.}

In this section, we are interested in the aspect ratios where the cavity is short (which means larger $h \delta$ ) in order to explore the so-called frustrated regime described by Chevalier et al. [6]. We present first the results on the stress components. We then compare the yielded boundary layer with [6] and study its width as a function of $B$.

\subsection{Stresses}

Figures 10 and 11 show every component of the stress tensor and its deviatoric part. We denote by $\tilde{\sigma}=-\tilde{p}$ Id $+\tilde{\tau}$ the whole stress tensor (recall that $\tilde{\tau}_{y y}=-\tilde{\tau}_{x x}$ and $\tilde{\sigma}_{x y}=\tilde{\tau}_{x y}$ ). We remark that we consistently find a value of $\|\tilde{\tau}\|$ which tends to $B$ close to the unyielded zones. We also remark the linear decrease of $\tilde{\tau}_{x y}$ along the $\tilde{y}$ axis in the entry/exit channel, which is also consistent with the Poiseuille flow theory. However, in the cavity, $\tilde{\tau}_{x y}$ along the $\tilde{y}$ axis is quasi-symmetric w.r.t to the middle of the yielded zone, which is reminiscent of the behaviour of $\partial_{y} \tilde{u}$ (see also figure 14, Right). These results are also in line with those of [28].

In Appendix A.5, we give other figures and details of pressure drops in the $\tilde{x}$ direction showing that the pressure is quasi-linear in the cavity.

We now show in more details the average pressure gradients inside the domain. First of all, we want to study the behaviour of the pressure in the inlet and outlet parts of the channel. In these zones, we expect $\partial_{x} \tilde{p}$ to be very close to the pressure gradient of the theoretical Poiseuille flow, denoted as $\partial_{x} \tilde{p}_{\mid t h}$. With notations of figure 1 , let us define (we drop the $\tilde{y}$ variable in $\tilde{p}(\tilde{x}, \tilde{y})$ since we recall we are on a horizontal cut, see Appendix A.5):

$$
D P_{\text {ext }}=\frac{\left|\tilde{p}\left(\tilde{L}_{\text {inout }}\right)-\tilde{p}(0)\right|}{\tilde{L}_{\text {inout }}} \text { and } D P_{\text {int }}=\frac{\left|\tilde{p}\left(\tilde{L}_{\text {inout }}+1 / \delta\right)-\tilde{p}\left(\tilde{L}_{\text {inout }}\right)\right|}{1 / \delta} .
$$

Figure 12 (resp. 13) shows the comparison between $D P_{\text {ext }}, D P_{\text {int }}$ and $\partial_{x} p_{\mid t h}$ determined as a function of $B$, for $\delta h=1$ (resp. $\delta h=0.5$ ). We observe that $D P_{\text {ext }}$ matches quite well with $\partial_{x} p_{\mid t h}$. In addition, as seen in figure 29, it appears that the average pressure gradient in the cavity is much lower than in the entrance.

\subsection{The boundary layer}

In figure 10, we can observe a whole domain in the back of the cavity where there is no flow, the already mentioned dead zone. Between the central plug and this dead zone, we can see a zone where the material deforms and behaves like a fluid: following Chevalier et al. [6], we will from now on call it the boundary layer. Velocity profiles in the cavity are displayed in figure 14. One can notice that near the middle of the cavity, the profiles overlap well each other, as noticed in [6]. We also retrieve the same kind of decrease and increase of the maximum velocity when passing by the cavity. Remark that velocity profiles seem to intersect in the same point, a point which will be explored in more details in section 4.

As mentioned in [6], we can also see that - contrary to the uniform flow in a conduit where the shear rate varies from 0 along the plug to its maximum value at the wall - the shear rate is not monotone in the boundary layer. This is illustrated in figure 14 (right), in agreement with the inflection point exhibited by the corresponding velocity profile of 

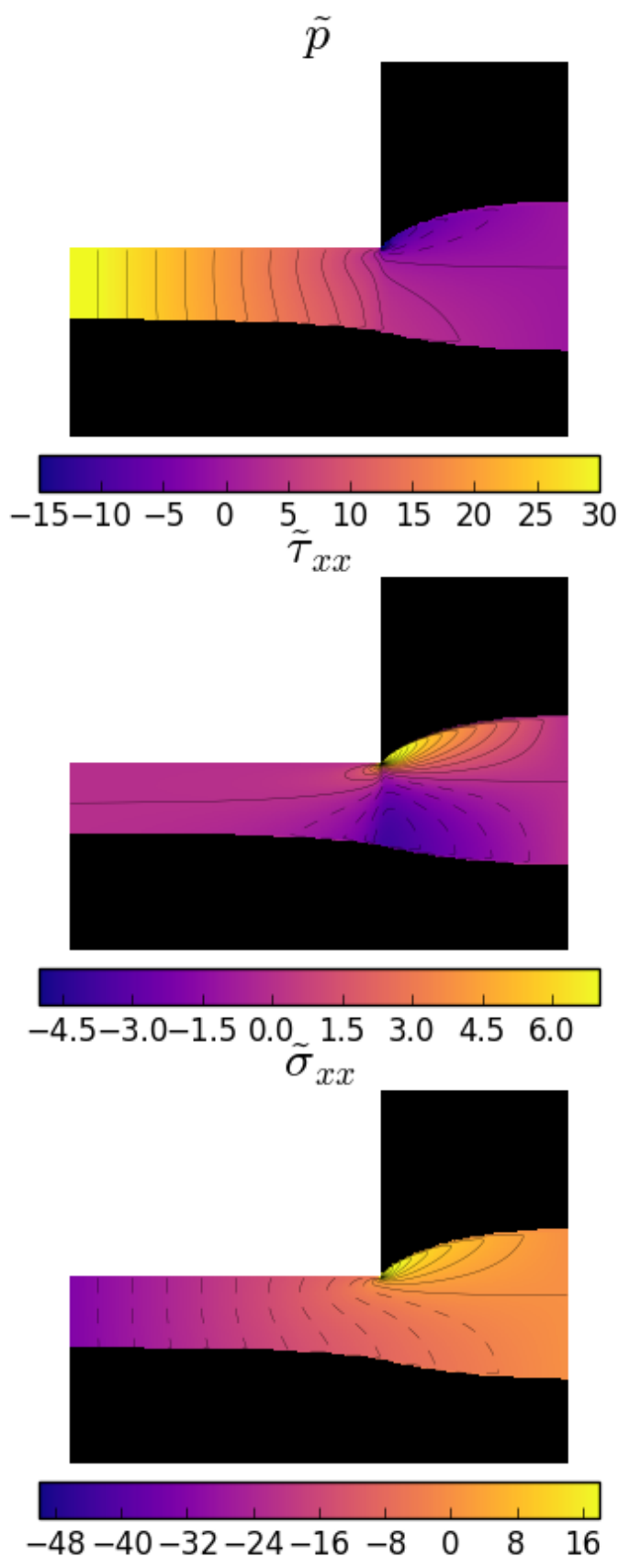
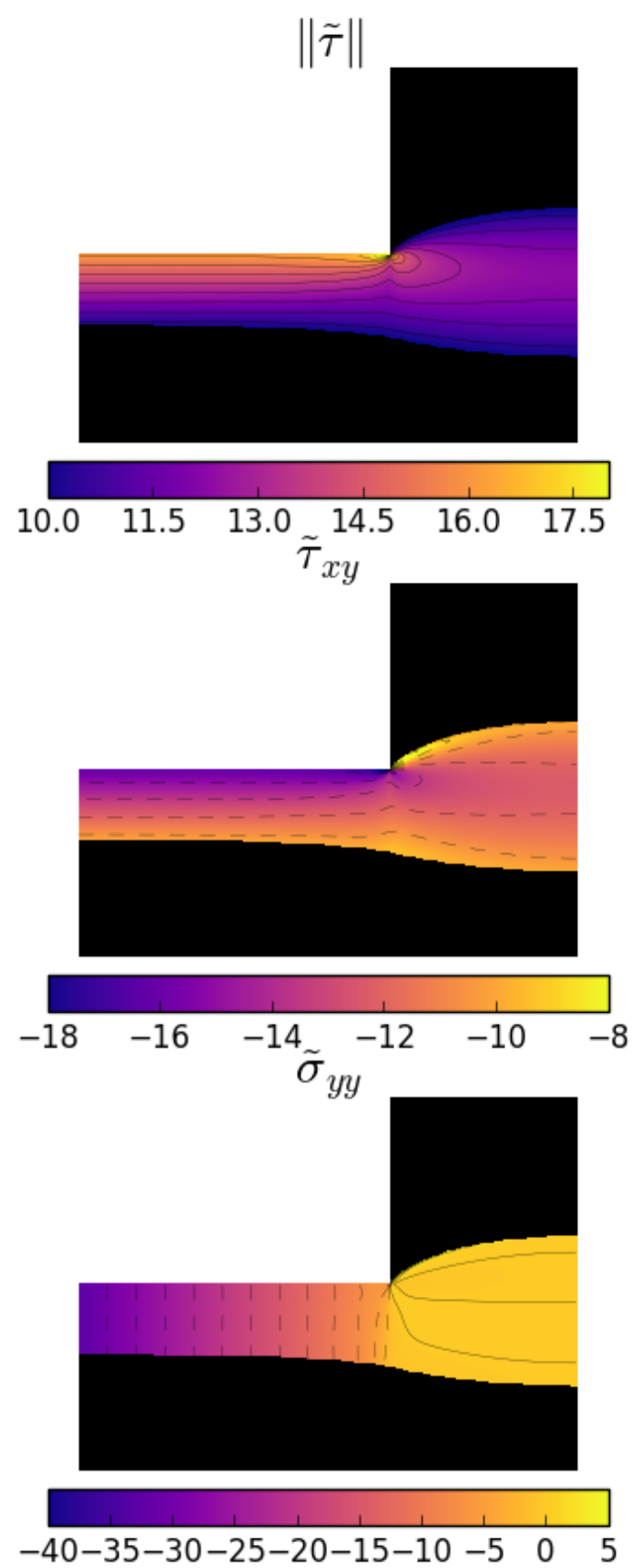

Figure 10: Stress tensor for $\delta=0.5, h=2$ and $B=10$ near the cavity entrance. Top: $\tilde{p}$ (left) and $\|\tilde{\tau}\|$ (right). Middle: $\tilde{\tau}_{x x}$ (left) and $\tilde{\tau}_{x y}$ (right). Bottom: $\tilde{\sigma}_{x x}$ (left) and $\tilde{\sigma}_{y y}$ (right).

figure 14 (left).

We now show the behaviour of the simulated width of the boundary layer as a function of $B$. Of course, when $B$ tends to 0 , the viscoplastic part of the material tends to disappear, the plug zone shrinks and finally disappears. We retrieve the fact that the boundary layer's width tends to $h$. Also, when, $B$ goes to infinity, the material approaches the fullplug/blocked behaviour and the boundary layer's width tends to 0 . What Chevalier's $\mathrm{PhD}$ thesis pointed out was a law of decrease of this width when $B$ tends to infinity. We present in figure 15 (right) an example of such evolution of this boundary layer width in a particular geometry. We also retrieve the fact (see figure 15, left) that the velocity 

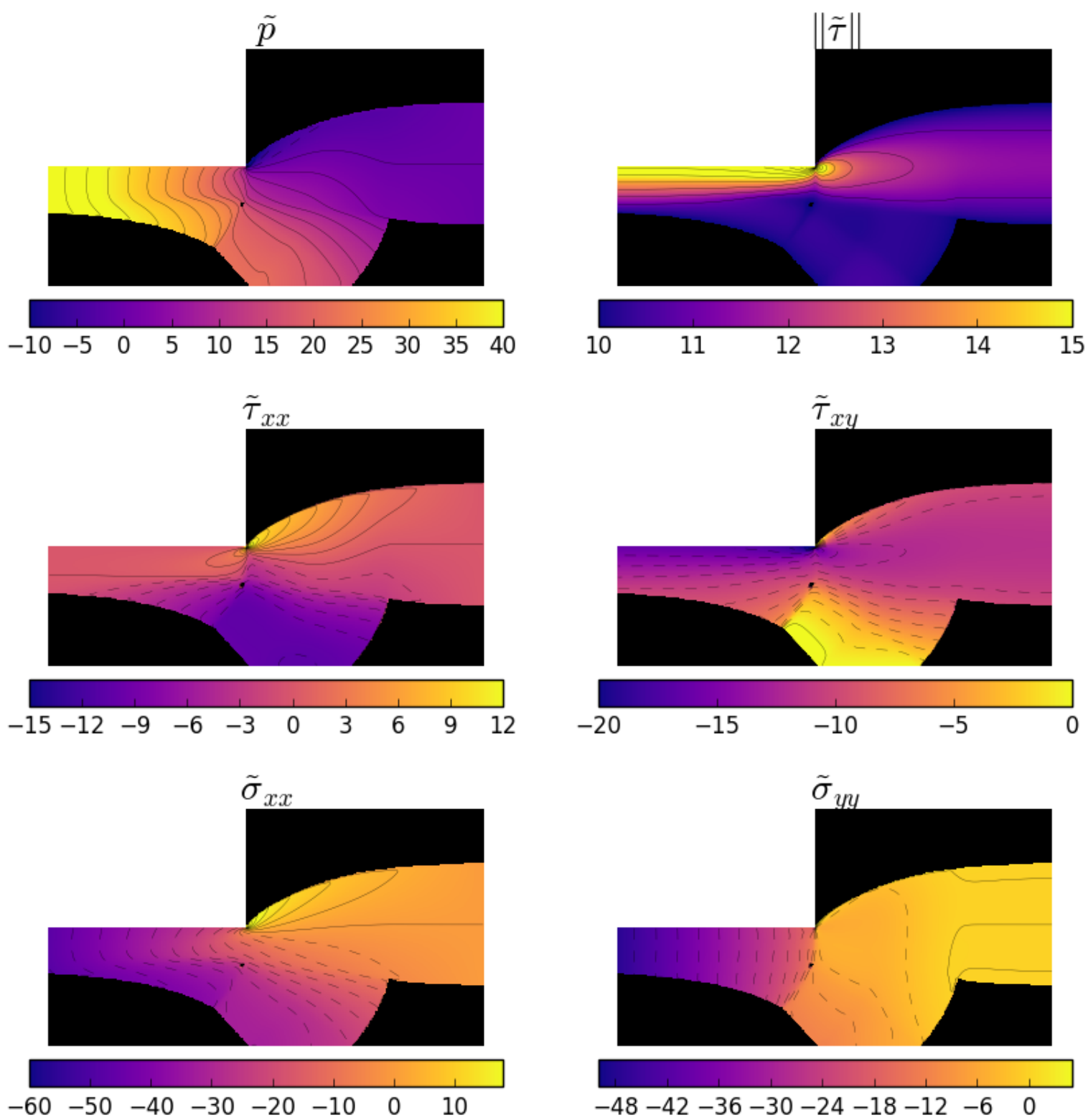

Figure 11: Stress tensor for $\delta=0.25, h=2$ and $B=10$ near the cavity entrance. Top: $\tilde{p}$ (left) and $\|\tilde{\tau}\|$ (right). Middle: $\tilde{\tau}_{x x}$ (left) and $\tilde{\tau}_{x y}$ (right). Bottom: $\tilde{\sigma}_{x x}$ (left) and $\tilde{\sigma}_{y y}$ (right).

profiles are close one another at high $B$ and tend to the function $y \mapsto \mathbb{1}_{[-1,1]}(y)$.

It seems that a law of decrease of this width $W$ is $W=\alpha B^{\beta}$. We present in figure 16 a tentative of linear fit in the log-log scale for two sets of $\delta$ and $h$ which gives slopes of -0.348 and -0.315 . These values are to be compared with the one of [6, Fig. 3] which is -0.2 (for a Herschel-Bulkley fluid). Remark that for a Bingham model, Oldroyd [23] showed that the yielded boundary layer is of order $B^{-1 / 3}$. It appears that $W$ is close to this scaling, even if the present configuration of frustrated geometry is not exactly the same as in the Oldroyd hypotheses. Let us finally notice that the central plug tends monotonically to 1 as $B \rightarrow \infty$ and tends to zero when $B \rightarrow 0$ (which is consistent with the Newtonian limit $B=0$ ). 


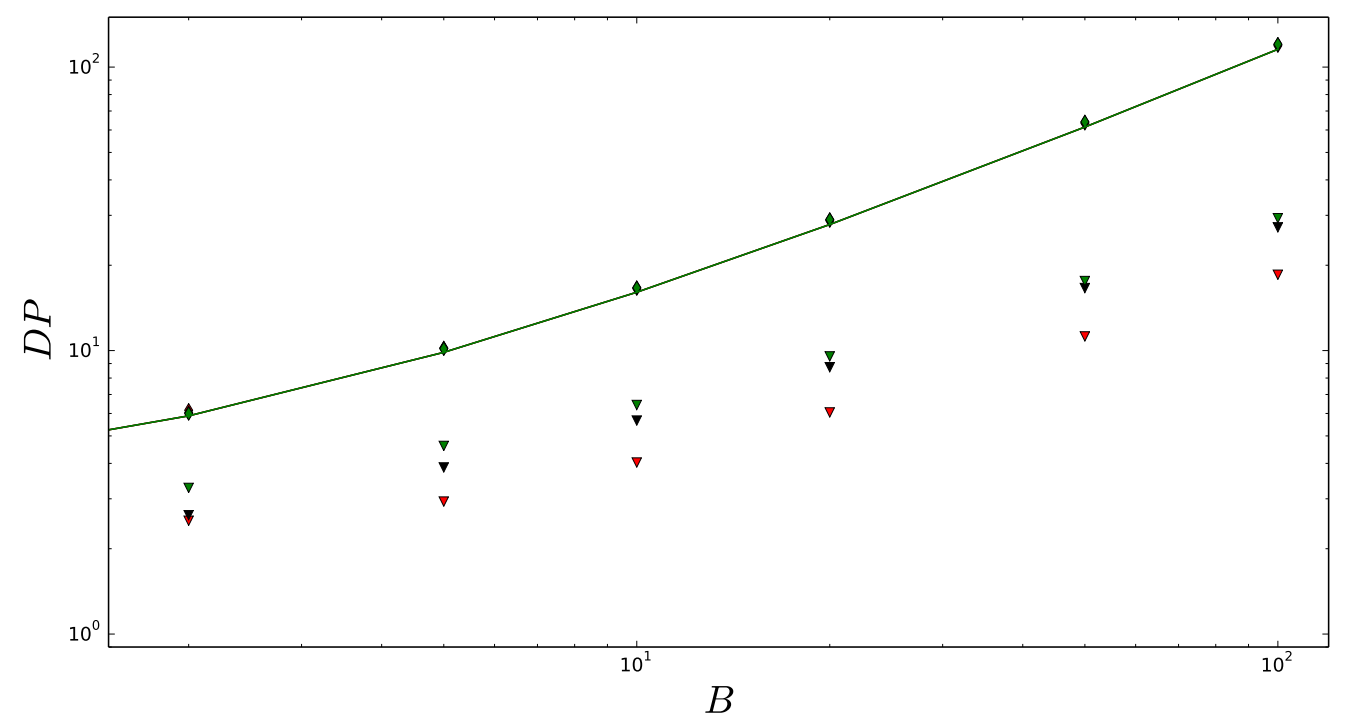

Figure 12: Study of the average pressure gradients for $\delta h=1$. Red (resp. black and green) glyphs represent $h=2$ (resp. $h=6 / 5$ and $h=12 / 11$ ). Quadrangles correspond to $D P_{\text {ext }}$, triangles to $D P_{\text {int }}$. Continuous curves corresponds to pressure gradient of the Poiseuille theory.

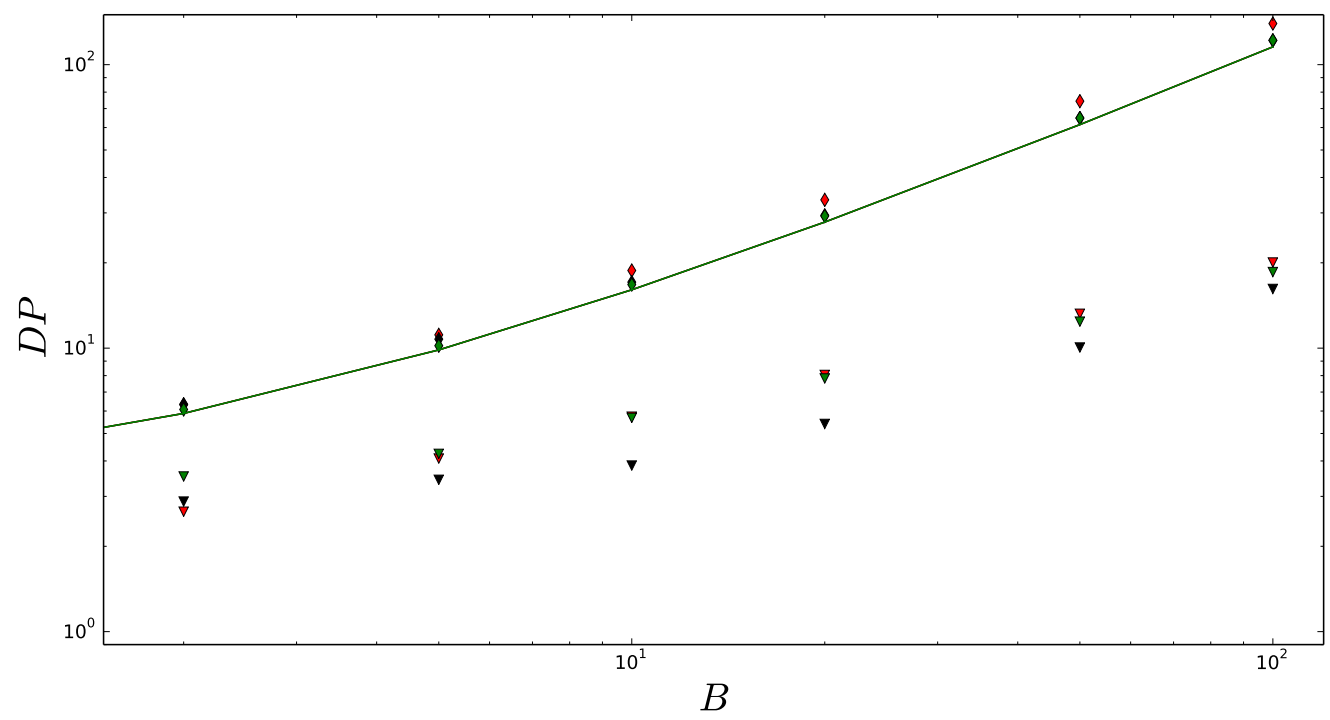

Figure 13: Study of the average pressure gradients for $\delta h=0.5$. Same glyphs and colors as the previous figure. 

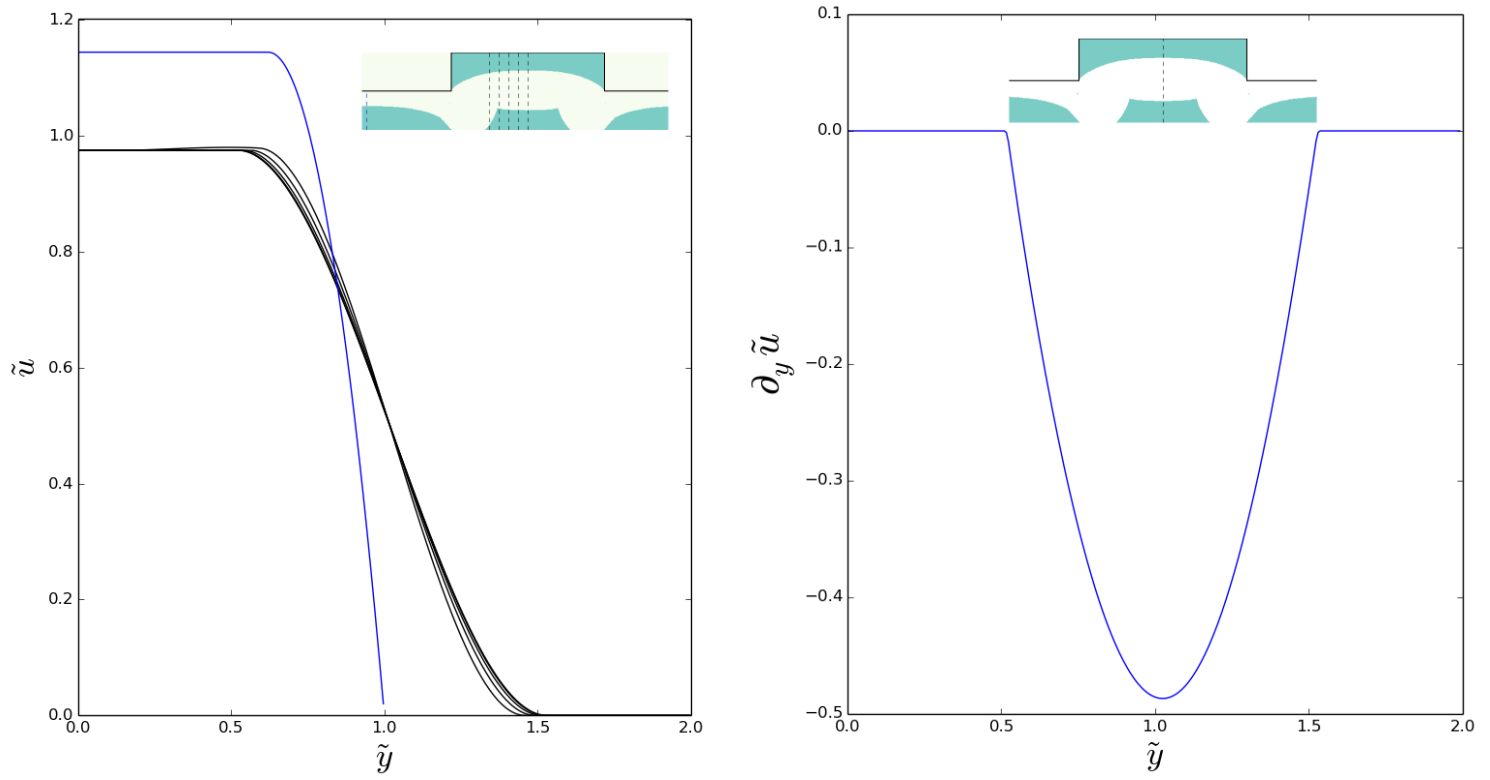

Figure 14: Left: different velocity profiles (in black) along the dashed lines shown on the inset, for $\delta=0.25, h=2$ and $B=10$. In blue, the profile far upstream. Right: $\partial_{y} \tilde{u}$ for the section in the middle of the cavity (shown by dashed line on the inset). Results of [6] are retrieved.
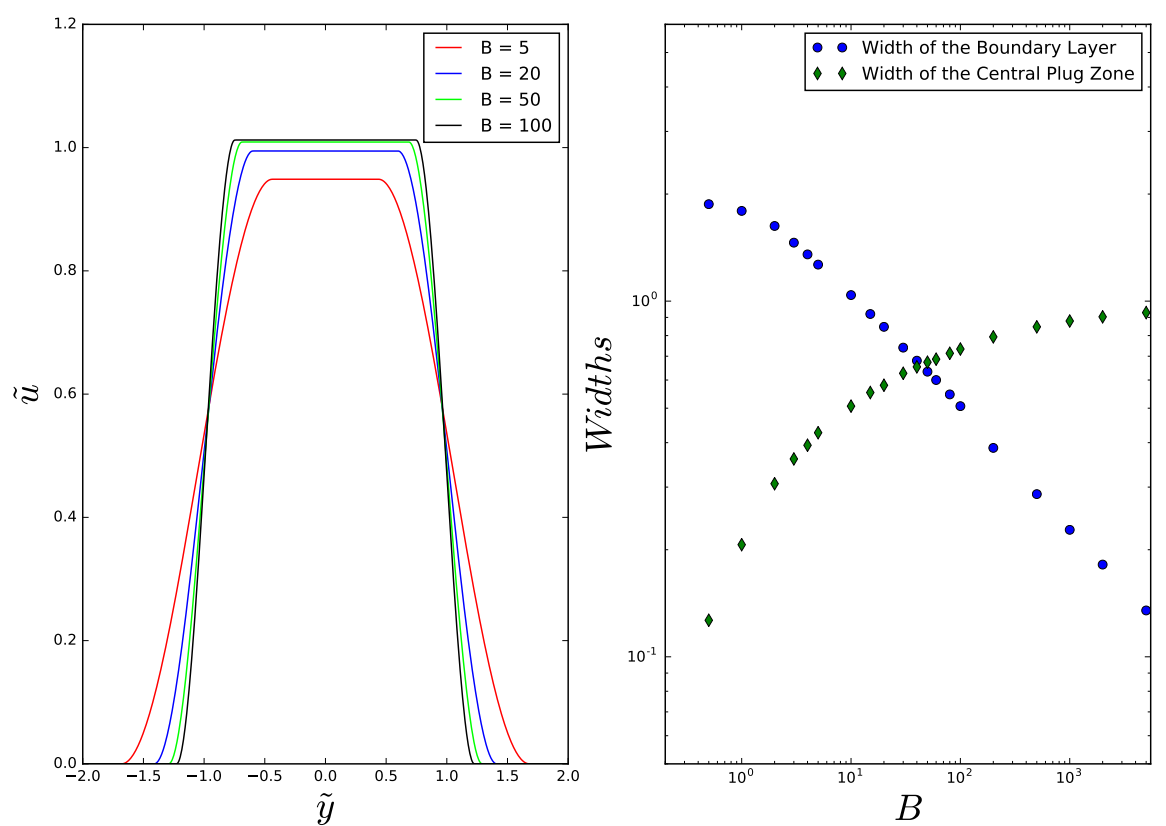

Figure 15: Left: superposition of velocities in the middle of the cavity for different $B$ with $\delta=0.25$ and $h=2$. Right: Widths of the boundary layer and dead zone as a function of $B$. Results of [6] are retrieved. 

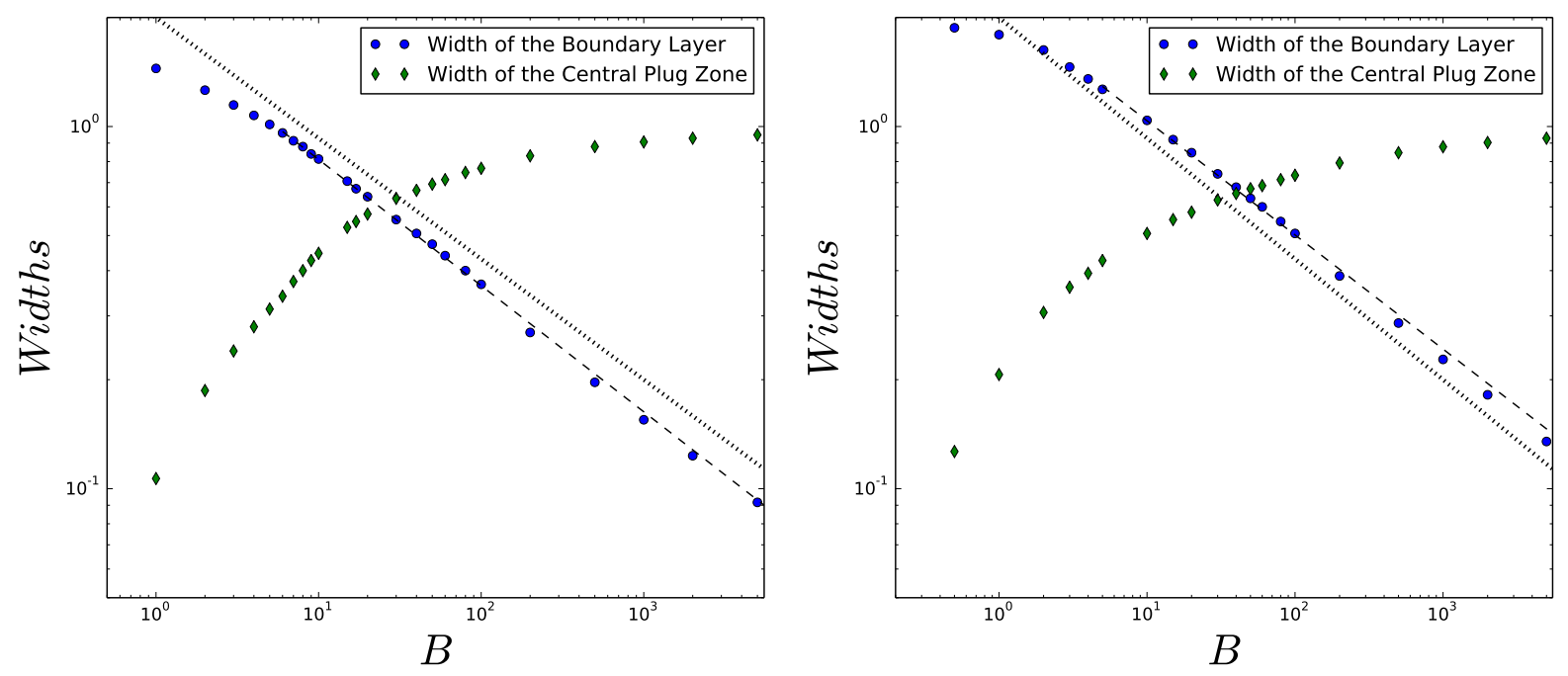

Figure 16: Different widths of boundary layers and dead zones with linear fit attempt (dashed lines) for $h=2, \delta=0.5$ (left) and 0.25 (right). The slope of the dashed lines are -0.348 and -0.315 , respectively. The Oldroyd's scaling of $B^{-1 / 3}$ is shown with dotted lines (see text). 


\section{Experiments of Luu et al.}

In this section, the objective is to mimic the experiments of Luu et al. [19] of a Carbopol flow over a step. We study how a Bingham model can reproduce the features shown in [19]. Therefore, we choose to present the results in a dimensional form. In the simulations presented in this section, $H=1, D=5$ and $L$ is equal either to 25 or 60 . We set the fluid properties equal to the ones of [19]: $\eta=1.84 P a \cdot s$ and $\tau_{y}=1.36 P a$. Note that here, the only free parameter of the flow is $B$, determined by the pressure gradient we impose in the entrance.

In Appendix A.4, the interested reader will find the corresponding strain rate fields in the same way as in Figure 3, to have details of the pseudo-plugs.

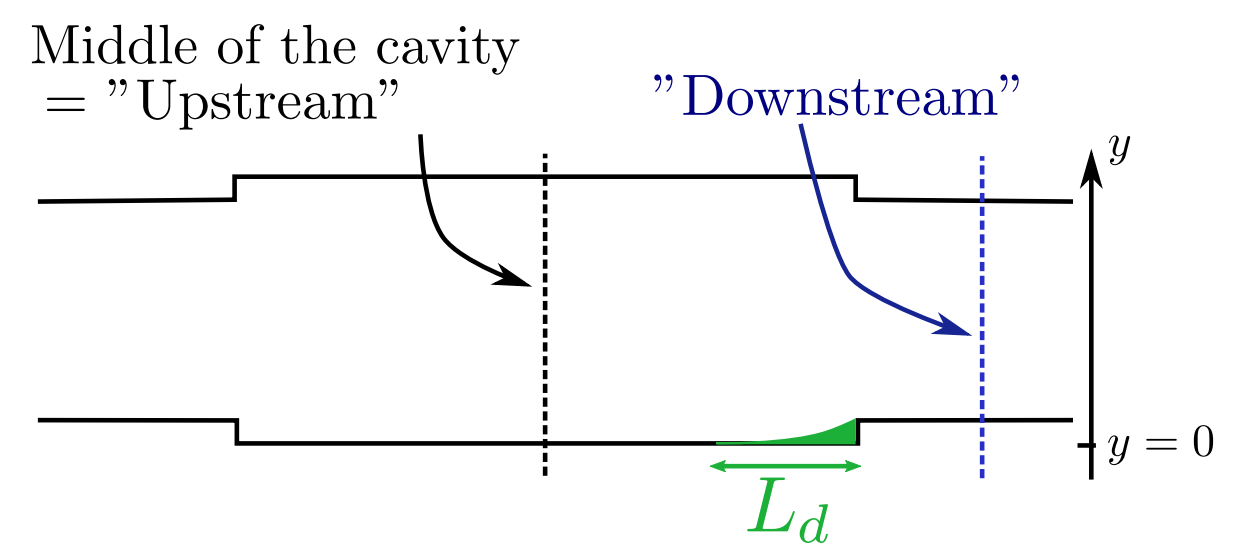

Figure 17: A typical aspect ratio for longer channels. Definition of the "Upstream" and "Downstream" cuts for velocity profiles of section 4.1. A typical dead zone in the configuration of [19] is shown in green at the corner of the exit step and gives the definition of the length of the dead zone $L_{d}$.

\subsection{Up and Downstream Poiseuille checks}

We will use a different origin ( 0 is the bottom boundary and not the middle of the channel), see figure 17 . For a channel of half-width $D$, the Poiseuille flow is:

$$
u(y)= \begin{cases}u_{\text {plug }}\left(1-\left(1-\frac{y}{y_{\text {plug }}}\right)^{2}\right) & \text { if } 0 \leqslant y \leqslant y_{\text {plug }} \\ u_{\text {plug }}=\frac{\left|\partial_{x} P\right|}{2 \eta} y_{\text {plug }}^{2} & \text { if } y_{\text {plug }}<y<D .\end{cases}
$$

where $y_{p l u g}$ is now defined as the beginning of the plug zone and $\partial_{x} P$ is the driving pressure gradient. Equation (8) becomes in the fluid part:

$$
\left(\frac{u_{\text {plug }}-u(y)}{u_{\text {plug }}}\right)^{1 / 2}=1-\frac{y}{y_{\text {plug }}},
$$

leading to a linear graph for $\sqrt{u_{p l u g}-u}$ as a function of $y$. In other words, a perfect Poiseuille flow should read:

$$
\left(\frac{u_{\text {plug }}-u(y)}{u_{\text {plug }}}\right)^{1 / 2}=\left\{\begin{aligned}
& 1-\frac{y}{y_{p l u g}} \text { if } 0 \leqslant y \leqslant y_{\text {plug }} \\
& 0 \text { if } y>y_{\text {plug }} .
\end{aligned}\right.
$$


Here, the cavity is long enough in order to recover a Poiseuille flow in its middle. Indeed, figure 18 (left) shows the superposition of the computed velocity profiles and the associated theoretical Poiseuille flows (in dashed lines) for different cavity lengths. We have also chosen to show (on the right) $\left(\frac{u_{\text {plug }}-u}{u_{\text {plug }}}\right)^{1 / 2}$ as a function of $y / y_{\text {plug }}$ : it can be seen that curves collapse quite well on the single line $y=1-x$ in the fluid part, as shown in (9). Even if the longest cavity $(L=60)$ provides a better approximation of the required flow, both figures are very similar. To save computation time, we will use the smaller cavity $L=25$, since it still allows to set up a Poiseuille flow and to recover the analysis of [19].
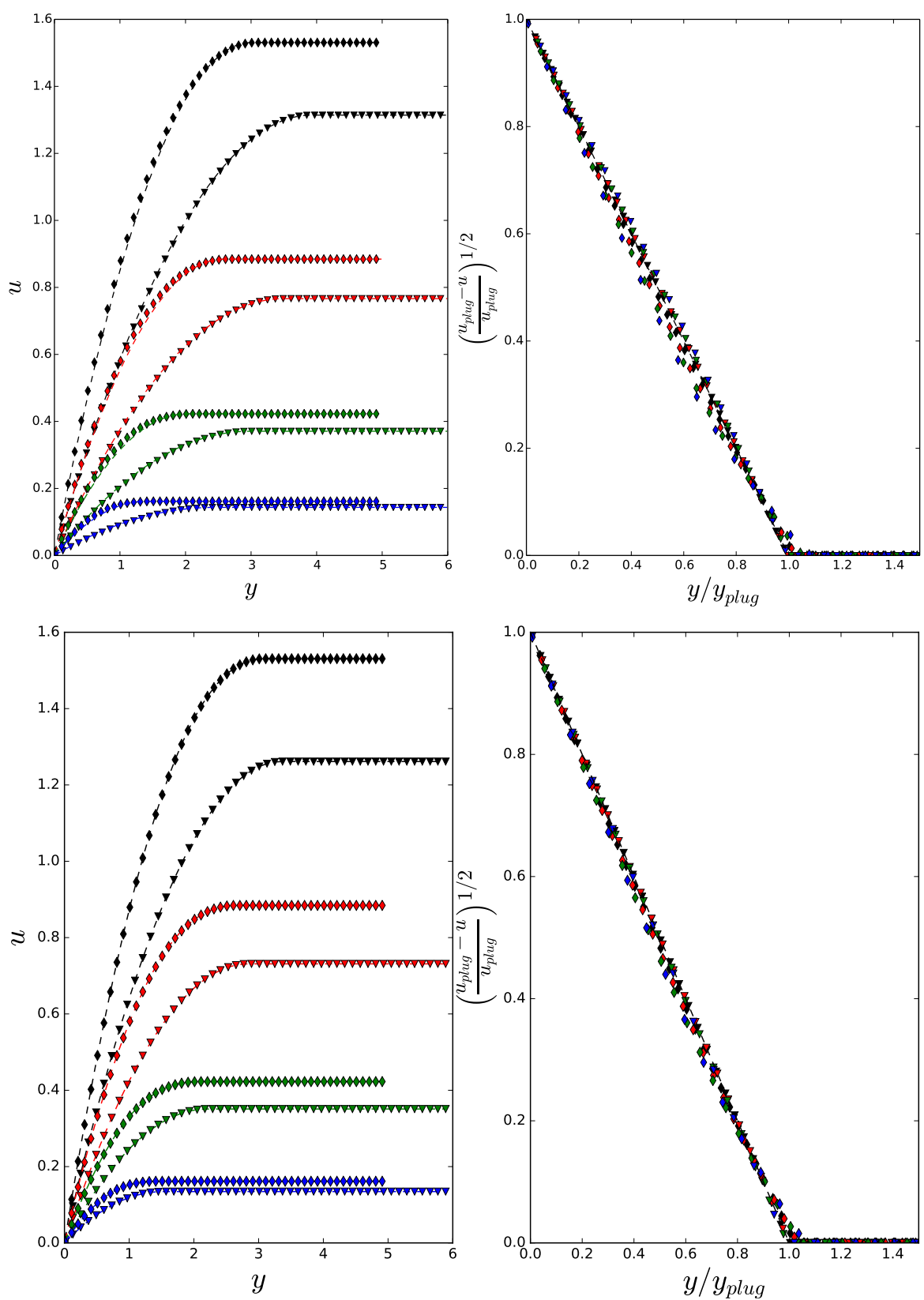

Figure 18: Velocity profiles in the middle of the cavity (triangles) and downstream (quadrangles) for $L=25$ (top) and 60 (bottom) and $B=3$ (black), 5 (red), 10 (green) and 25 (blue). Left, the dashed lines represent the theoretical Poiseuille profile for the associated $u_{p l u g}$ and $y_{p l u g}$. Right: collapsing of the curves following (9); the dashed line is an indication for the eye. 


\subsection{The slip line}

In this section we take interest in what happens closer to the exit step. Of course the presence of the step will deviate the streamlines from their initial horizontal direction, to force them to re-enter the exit channel. In [19], the authors outlined the fact that the streamlines were almost parallel far from the dead zone and far from the step corner. Consequently, they investigate the velocity in a frame tilted by an angle $\theta$ and find that velocity profiles all intersect at the same point $\left(y_{s}, u_{s}\right)$. In fact, this phenomena can be seen for various values of the angle $\theta$. As figure 19 shows, if $\theta$ is small enough, (small in order to let the $x$-axis in the dead zone), we can still observe an intersection point, the only change being the value of $y_{s}$ and $u_{s}$. In the following, we choose for $\theta$, the angle between the tangent of the dead zone and the wall, at their point of intersection.
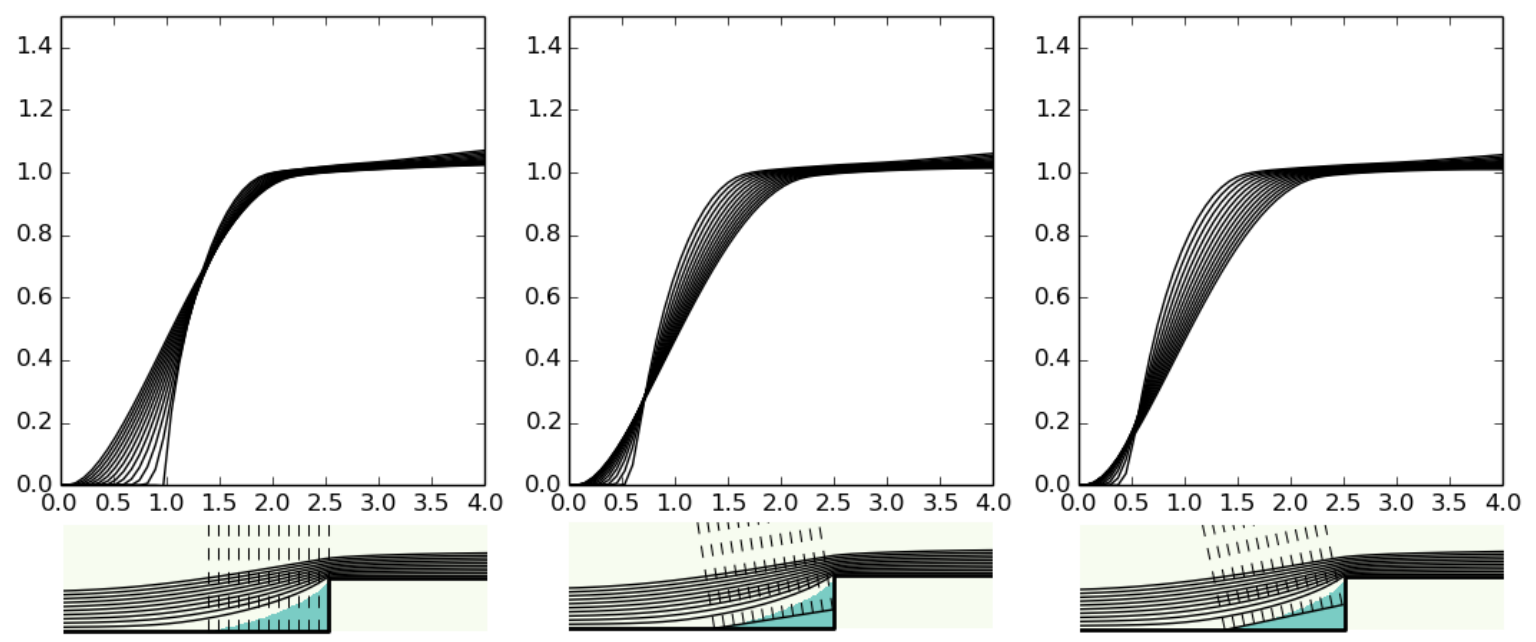

Figure 19: Different angles $\theta$ (from left to right: $0, \pi / 20, \pi / 15$ ) of the tilted frames for $L=25$ and $B=20$. Velocity profiles (rescaled by the averaged inlet velocity) taken as shown on the bottom diagrams.

We retrieve the fact that all these profiles intersect at a same point $\left(y_{s}, u_{s}\right)$. The line $y=y_{s}$ in the tilted frame is called the slip line in [19]. Along this line $u$ is constant equal to $u_{s}$, which means that, in the tilted frame, $\frac{\partial u}{\partial x}$ is 0 . This does not mean of course that the material behaves rigidly since $\frac{\partial u}{\partial y}+\frac{\partial v}{\partial x}$ is not zero but it gives us a hint to decompose the flow in two zones: above and below the slip line.

On figure 20 are shown some velocity profiles for different $B$ and the associated point $\left(y_{s}, u_{s}\right)$. Below this point, the frame meets the dead zone. Far above this point, a plateau can be observed, the values of the plateau increasing as the material approaches the step. In the following, we focus our study on just one profile, the one in the middle of the tilted segment (the red dashed line in figure 20, left). We take interest in the profile above the slip line and we will try to see whether it is close to a Poiseuille profile.

From this point to the end, since there is not a real plateau, we define $u_{\text {plug }}$ as the averaged velocity between $y_{p l u g}$ and the end of the section. Therefore, we define a pseudoplug line as follows. We first make a rough linear fit of the slope of the linear part of the profile. We then define $y_{p l u g}$ as the intersection of the linear part with the line $u-u_{\text {plug }}=0$. Note that this would have been the exact plug location if we have had a real Poiseuille flow above $y_{s}$. On figure 21, we show the reconstructed $y_{s}$ and $y_{p l u g}$ lines, following [19, Fig. 8], for various $B$ : we recover the same qualitative behaviour as in [19].

In the sequel, we fit $\left(\frac{u_{p l u g}-u(y)}{u_{\text {plug }}}\right)^{1 / 2}$ with a line on the segment $\left[y_{s}, y_{f i t}\right], y_{f i t}$ being the 

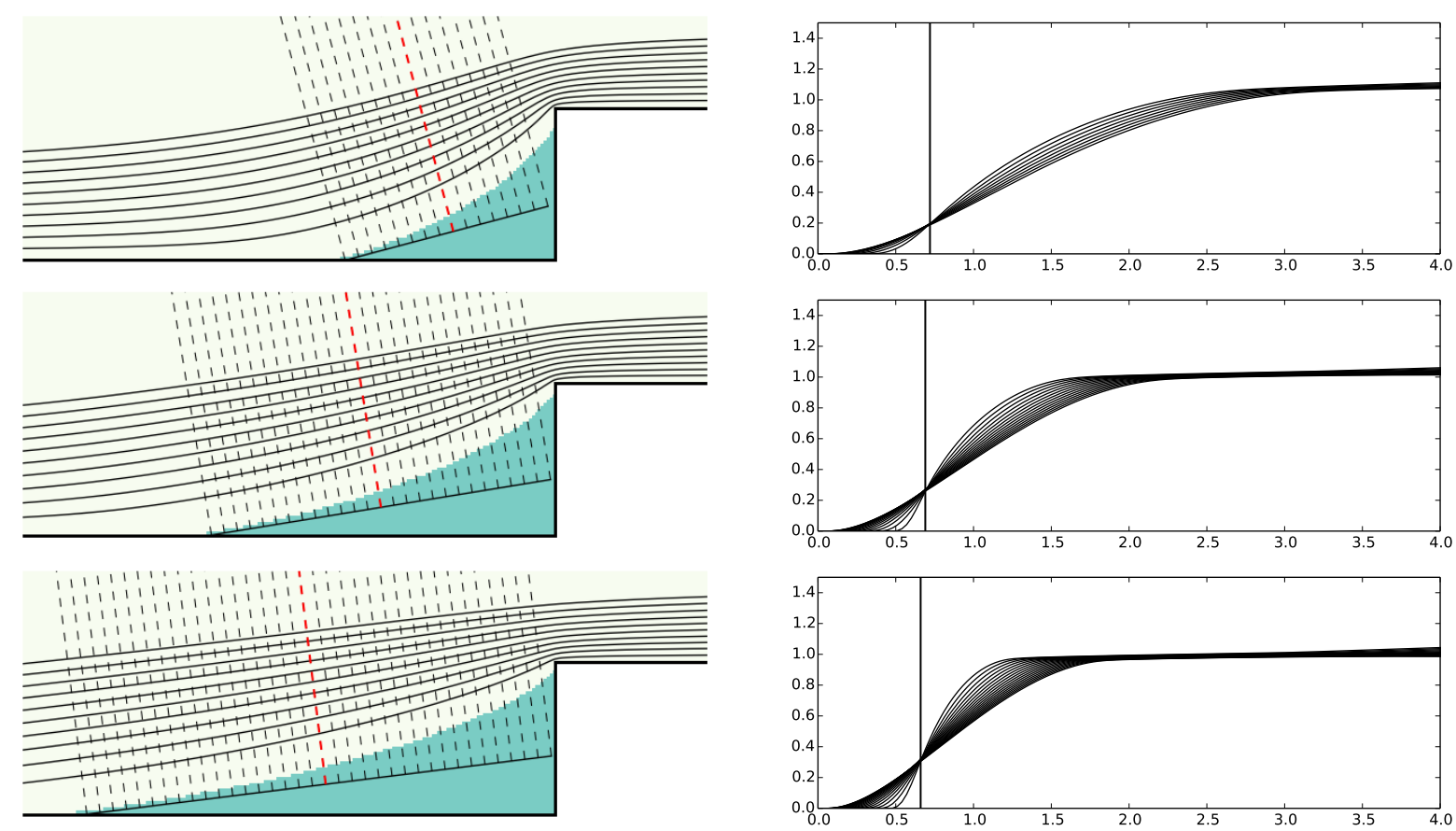

Figure 20: Existence of $\left(y_{s}, u_{s}\right)$ for various $B$ : from top to bottom $B=5,20,50$. Left: streamlines (black lines), dead zone (dark green in the corner of the step), probe lines (dashed dark lines) for the velocity profiles shown on the right. The dashed red line is used in the study of Fig. 22. Right: velocity profiles (rescaled by the averaged inlet velocity) and localization of $y_{s}$.
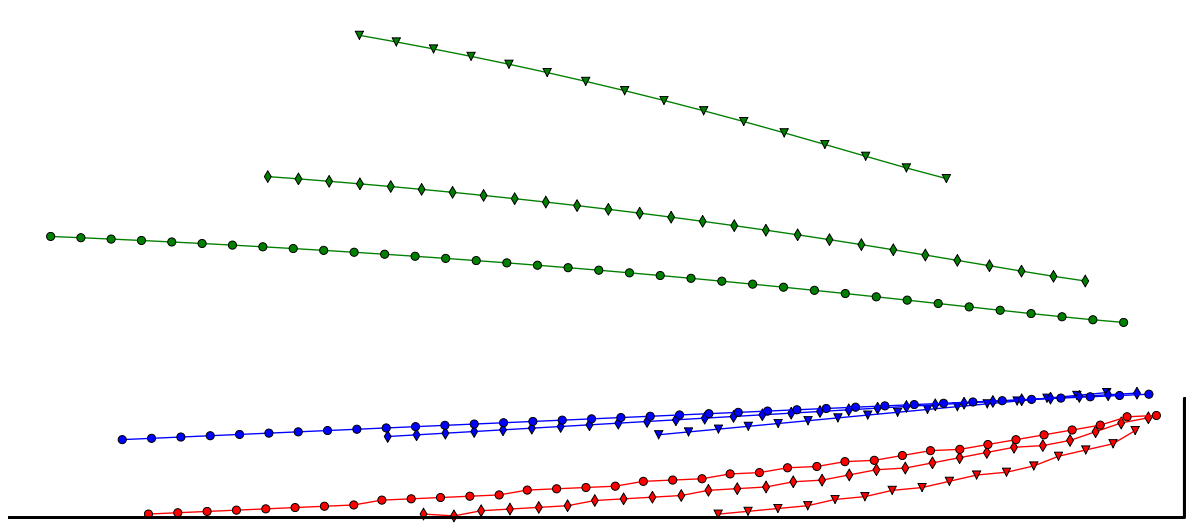

Figure 21: Different zones, inspired by [19]: dead zone (red), slip line (blue, based on $y_{s}$ ) and plug line (green, based on $y_{\text {plug }}$ ) for $B=5$ (triangles), 20 (quadrangles), 50 (circles).

beginning of the pseudo-plateau. Note that the pseudo-plateau $\left(y>y_{f i t}\right)$ and the pseudoplug $\left(y>y_{\text {plug }}\right)$ are two different domains. Figure 22 shows on top the linear fit we made (the errors are of the order $10^{-4}-10^{-6}$ ). The dashed red lines show the borders of the fit domain, $y_{s}$ and $y_{f i t}$, while the dashed blue lines show respectively (from left to right)

- $y_{\text {dead }}$ : the end of the dead zone, 
- $y_{i}$ : the maximum of the profile of $|d|$ as it is linked to the inflection point of the velocity,

- $y_{p l u g}$ defined as before.

We recover the results of [19], i.e. the fact that the material follows for a large part a Poiseuille flow near the exit step.
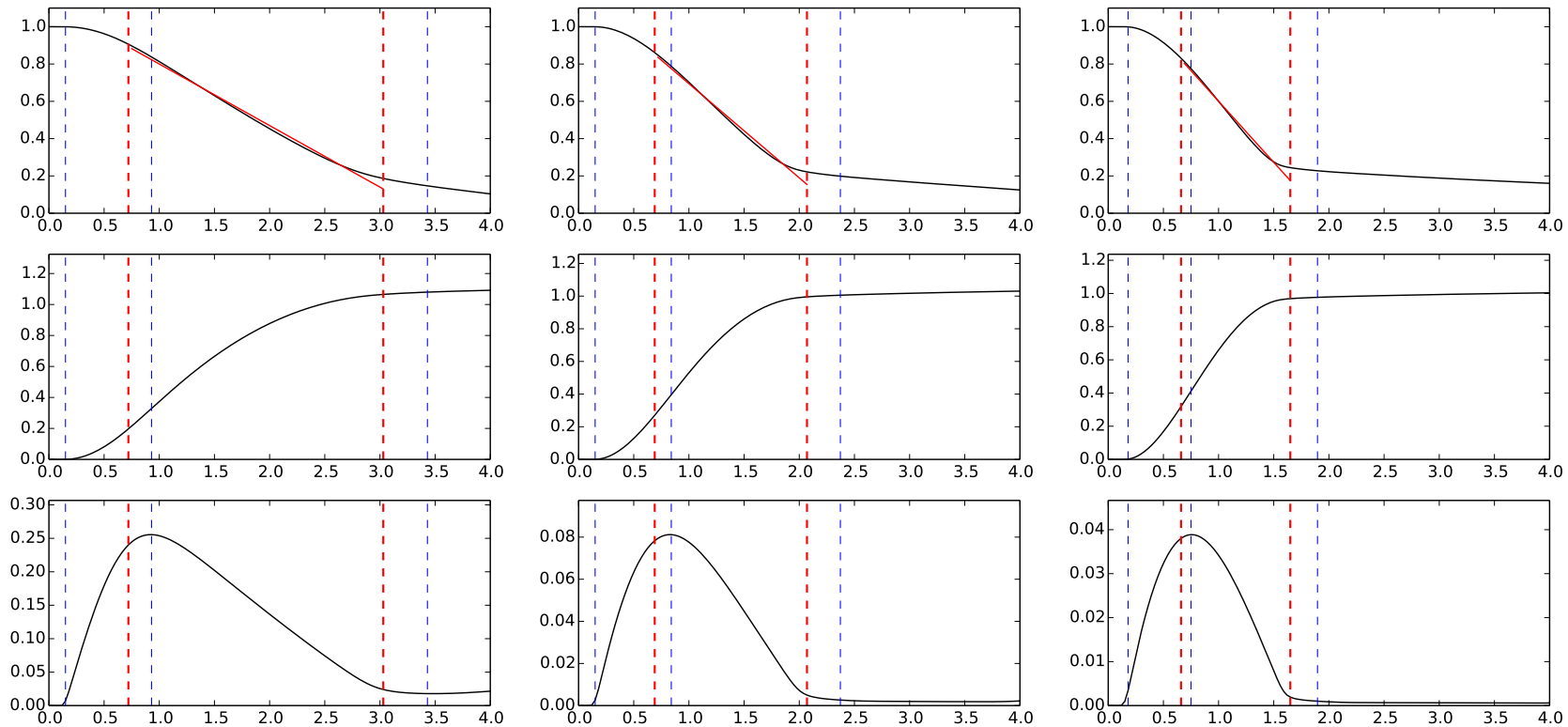

Figure 22: From top to bottom : $\sqrt{\frac{u_{p l u g}-u(y)}{u_{\text {plug }}}}$ and linear fit in the fluid part, $u$ and $|d|$, as a function of $y$, as in [19]. From left to right: $B=5,20,50$. Red dashed lines: borders of the linear fit domain. Blue dashed lines (from left to right): $y_{\text {dead }}$ (end of the dead zone), $y_{i}$ (maximum of $\left.|d|\right)$ and $y_{\text {plug }}$.

If we leave out the part below $y_{s}$, and suppose we have the slip velocity $u_{s}$, the equation (9) becomes

$$
\left(\frac{u_{\text {plug }}-u(y)}{u_{\text {plug }}-u_{s}}\right)^{1 / 2}=\left\{\begin{aligned}
& 1-\frac{y-y_{s}}{y_{\text {plug }}-y_{s}} \text { if } 0 \leqslant y \leqslant y_{\text {plug }} \\
& 0 \text { if } y>y_{\text {plug }},
\end{aligned}\right.
$$

Our numerical simulations also verify the fact that the exit flow above the slip line is consistent with the above Poiseuille theory given in [19]. Indeed, figure 23 shows many cuts of dimensionless velocities to the power $1 / 2$ in the tilted frame for different $B$. We can observe the collapse of all profiles on the same dashed line which is plotted using the theoretical prediction of the RHS of (10).

\subsection{Effective section}

Let us consider the Poiseuille flow for channel of width $D$. It has the following flow rate :

$$
Q_{p}=\int_{0}^{D} u(y) \mathrm{d} y \text { i.e. } u_{p l u g}=\frac{Q_{p}}{D-y_{p l u g} / 3} .
$$

This means that the flow is equivalent to a uniform one passing through a smaller section of width $D-y_{p l u g} / 3$. If we consider only the upper part of the flow, i.e. above $y_{s}$, we can 


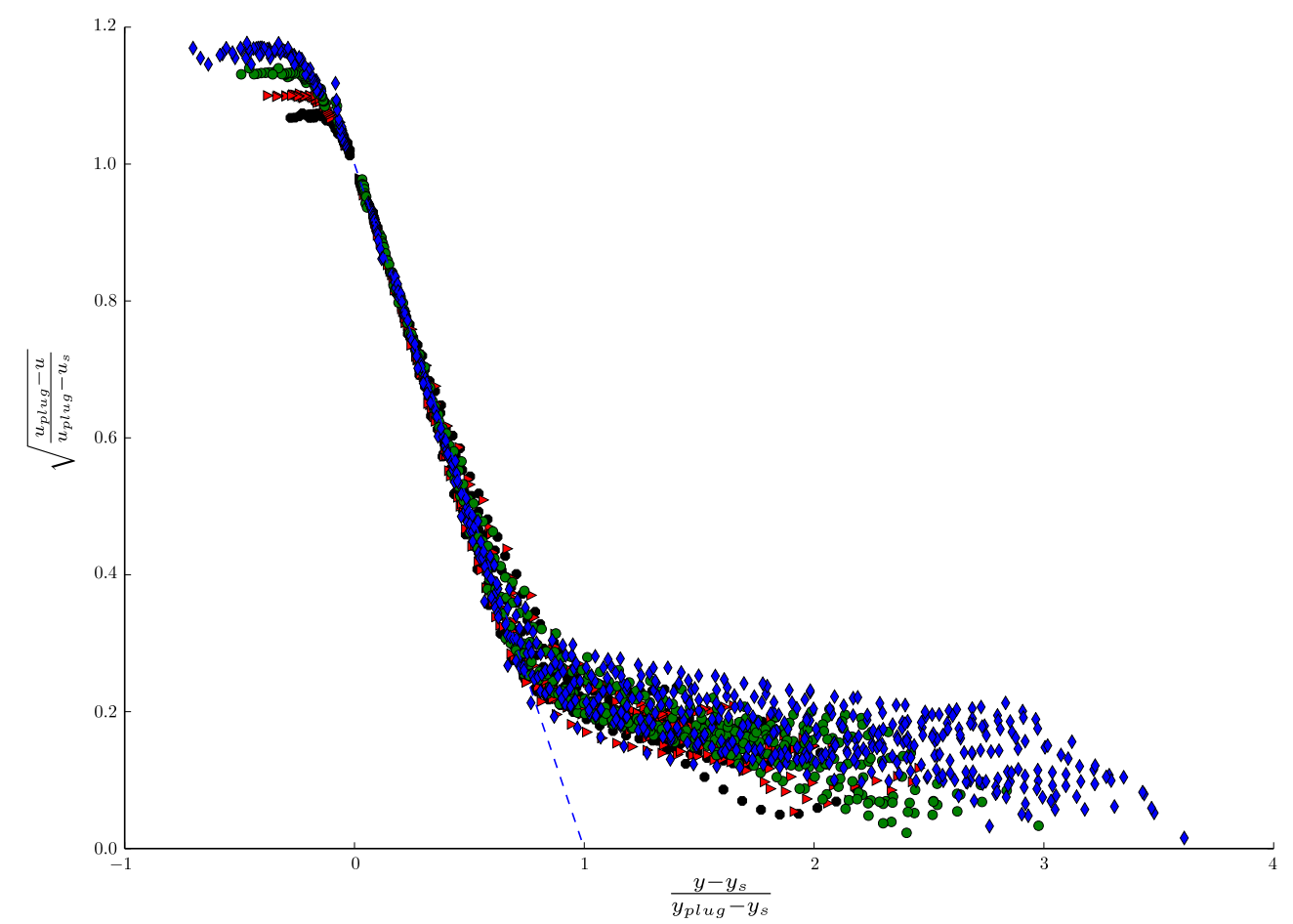

Figure 23: Rescaling of the simulated velocity profiles using the LHS of (10). The dashed line represents the theoretical prediction of the RHS of (10).

define the effective flow rate, which is :

$$
Q_{e f f}=\int_{y_{s}}^{D}\left[u(y)-u_{s}\right] \mathrm{d} y
$$

As before, we can compute $u_{p l u g}-u_{s}$ as a function of $Q_{e f f}$, which is :

$$
u_{p l u g}-u_{s}=\frac{Q_{e f f}}{\left(D-y_{s}\right)\left(1-\left(y_{p l u g}-y_{s}\right) / 3\right)}=\frac{Q_{e f f}}{S_{e f f}} .
$$

Here, the denominator plays the same role as above, as this is an effective section through which the material passes. Another sign of the consistency of the velocity profiles with Poiseuille theory would be the numerical reconstitution of equation (11). That's what we find out in figure 24 with the fit of all points over the same line.

\section{Conclusion}

In this article, we presented a numerical approach based on a coupling between finitedifferences for the discretization in space and Augmented Lagrangian methods to compute the solution of Bingham flows in an expansion-contraction geometry. The implementation is done with the MUMPS parallel solver allowing to make simulations with a significant number of mesh points (up to 7.8 million) within a reasonable CPU time (one or two days with 16 processors and using a quite demanding accuracy of at least $10^{-11}$ for the residue of the AL).

We made simulations in the configuration of Chevalier et al. [6]. After giving some insight on the stresses and average pressure gradients, we performed a detailed study of the dynamics of the boundary layer in the so-called frustrated regime. All the qualitative 


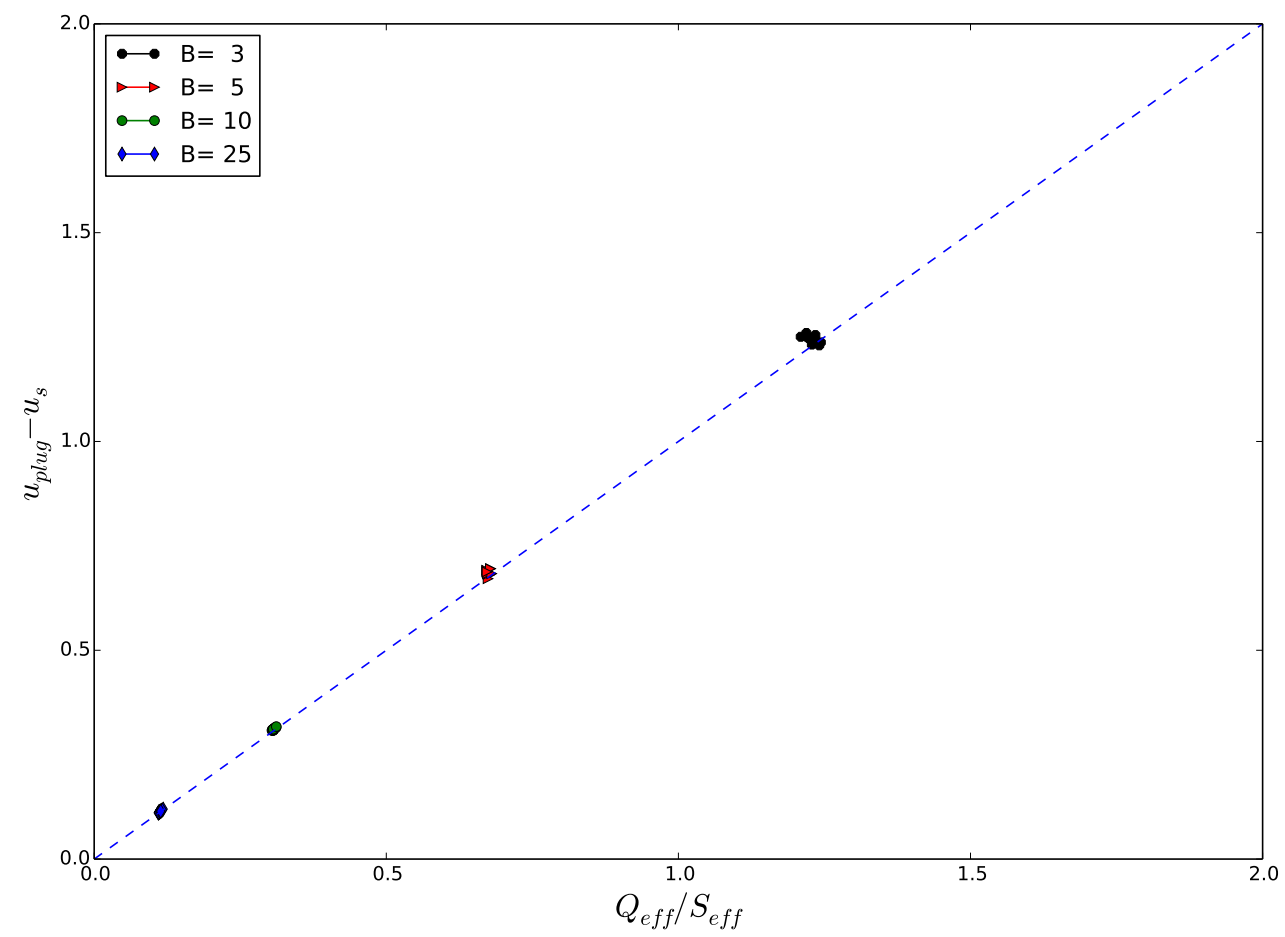

Figure 24: $u_{p l u g}-u_{s}$ as a function of $\frac{Q_{e f f}}{S_{e f f}}$. See equation (11) for more details.

features of [6] are retrieved: evolution of $u_{p l u g}$ passing by the cavity, superposition of dimensionless velocities at higher Bingham numbers, boundary layer thickness, etc. We presented the evolution of the width of this boundary layer and of the central plug (thus allowing also to compute the width of the dead zone in the cavity), as a function of the Bingham number: we find similar behaviour as the one presented in [6] and gave the corresponding exponent of the decreasing law in the case of a Bingham flow.

The accuracy of our simulations is then also successfully tested with the comparison on the physical experiments of Luu et al. [19]. Taking the Bingham case of their more general case based on Herschel-Bulkley, we retrieve numerically all their results. In particular, it appears that a Bingham constitutive law is also able to reproduce the slip line effect described in [19]. We showed that this phenomena is robust on the variation of the choice of the angle of the tilted reference frame. Finally, we exhibited a linear behaviour (in loglog coordinates) of the horizontal length of the dead zone as a function of the Bingham number, a test which can be used as a benchmark for future numerical studies.

\section{Acknowledgments}

We thank the Referees for their comments which contributed to improve the quality of the article. We are thankful to Philippe Coussot for stimulating discussions and for bringing us to the physical experiments studied in this article. We also thank Guillaume Chambon, Li-Hua Luu, Pierre Philippe and Didier Bresch for the fruitful interactions we can have in a common project dedicated to comparisons of physical experiments and numerical simulations of viscoplastic materials. This TelLUs project of INSU-INSMI (2016 Call) is funded by the CNRS, France. We thank all the MUMPS team, at LIP, ENS de Lyon, who taught us how to dive deep inside their solver, allowing to make the most of it.

Numerical simulations were done using computer resources of the Pôle Scientifique 
de Modélisation Numérique (PSMN), Lyon, France. We express all our gratitude to the PSMN staff for their flawless support allowing to smoothly port the code on the cluster. 


\section{A Details on the numerical methods}

\section{A.1 The Augmented Lagrangian algorithm}

We do not present the theory justifying the AL algorithm and refer the interested reader to [15]. We directly give the main steps of the AL algorithm for the solution of (1)-(2).

Algorithm 1 - Bingham problem (1)-(2):

- Initialization: affect any guess value for $p^{0}, d^{0}, \lambda^{0}$

- Repeat: $i \geqslant 1$

1. Generalized Stokes problem, solve for $\left(\mathbf{u}^{i}, p^{i}\right)$ :

$$
\begin{aligned}
-\nabla \cdot\left((r+2 \eta) D\left(\mathbf{u}^{i}\right)\right)+\nabla p^{i} & =\nabla \cdot\left(\lambda^{i-1}-r d^{i-1}\right) \\
\nabla \cdot \mathbf{u}^{i} & =0
\end{aligned}
$$

2. Explicit computation at each node of tensor

$$
d^{i}=\left\{\begin{aligned}
0 & \Leftrightarrow\left|\lambda^{i-1}+r D\left(\mathbf{u}^{i}\right)\right| \leqslant \tau_{y} \\
\frac{\lambda^{i-1}+r D\left(\mathbf{u}^{i}\right)}{r}\left(1-\frac{\tau_{y}}{\left|\lambda^{i-1}+r D\left(\mathbf{u}^{i}\right)\right|}\right) & \Leftrightarrow\left|\lambda^{i-1}+r D\left(\mathbf{u}^{i}\right)\right|>\tau_{y}
\end{aligned}\right.
$$

3. Update of the Lagrange multiplier $\lambda$ :

$$
\lambda^{i}=\lambda^{i-1}+r\left(D\left(\mathbf{u}^{i}\right)-d^{i}\right)
$$

- Until either $\left\|D\left(\mathbf{u}^{i}\right)-d^{i}\right\|_{L^{2}} \leq \operatorname{tol}_{B}$ or $i=N_{\max , B}$.

At convergence of Algorithm 1, we have a numerical solution of (1)-(2). To solve the Stokes problem (12), we use again an AL algorithm (see [11, 13]):

Algorithm 2 - Stokes problem (12):

- Initialization: affect $p^{i-1,0}=p^{i-1}$ (note the double exponent to remind the link with Algorithm 1) which is a natural guess coming from the "previous" iteration (in $i$, not $k$ )

- Repeat: $k \geqslant 1$

1. Solve the linear problem in $\mathbf{u}^{i-1, k}$ :

$$
-\nabla \cdot\left((r+2 \eta) D\left(\mathbf{u}^{i-1, k}\right)\right)-s \nabla\left(\nabla \cdot \mathbf{u}^{i-1, k}\right)=\nabla \cdot\left(\lambda^{i-1}-r d^{i-1}\right)-\nabla p^{i-1, k-1}
$$

2. Update the pressure (which is here a Lagrange multiplier):

$$
p^{i-1, k}=p^{i-1, k-1}-s \nabla \cdot \mathbf{u}^{i-1, k}
$$

- Until either $\left\|\nabla \cdot\left(\mathbf{u}^{i-1, k}\right)\right\|_{L^{2}} \leq t o l_{S}$ or $i=N_{\max , S}$.

- Set $\mathbf{u}^{i}=\mathbf{u}^{i-1, k}$ and $p^{i}=p^{i-1, k}$. 
Of note, this is an alternative approach compared to [32, 21] where an Uzawa/conjugate gradient algorithm (solving directly for $\left(\mathbf{u}^{i}, p^{i}\right)$ ) is used.

As shown in $[11,15], r$ and $s$ can be variable without preventing the algorithm to converge to the unique solution of (1)-(2). However, there exist values of these parameters which allow for a significantly smaller number of iterations in the respective AL loops. Except in rare cases, see e.g. [9], it is very difficult to derive a priori these optimal values. However, it is still possible to study them numerically for a given problem. In this paper, after extensive numerical tests on the concerned flows, typical values used are: $r=200$ and $s=2000$, since they are the values for which we solve the least linear systems. Of course these are the optimal values for a given geometry and for certain flow parameters. But it here gives the values close to the optimal values for the simulations of this paper.

In all the simulations presented in this article, we set $t_{o l}=5 \cdot 10^{-12}$. The value of $t_{0} l_{B}$ is $6 \cdot 10^{-12}$, unless otherwise stated. Of note, we did extensive tests with $\left(\right.$ tol $_{B}$, tol $\left._{S}\right)$ down to machine precision $\left(10^{-15}\right)$ : algorithms still converge but, since the quality of the results are not extremely more accurate than for the aforementioned values and are longer in CPU time, we do not perform all the tests with machine precision. We believe that $\left(\right.$ tol $_{B}$, tol $\left._{S}\right)=\left(6 \cdot 10^{-12}, 5 \cdot 10^{-12}\right)$ is still a quite demanding accuracy.

Concerning the maximal number of iterations, we put $N_{\max , B}=40000$ but in practice it is never reached except for long domains. For $N_{\max , S}$ we choose 1000 but in practice the number of iterations of Algorithm 2 does not exceed 200 for the first iteration of Algorithm 1 and then decreases quickly along the following iterations of Algorithm 1, reaching a typical value between 1 and 5 . This is illustrated in more details in section A.3.

\section{A.2 Spatial discretization and solver}
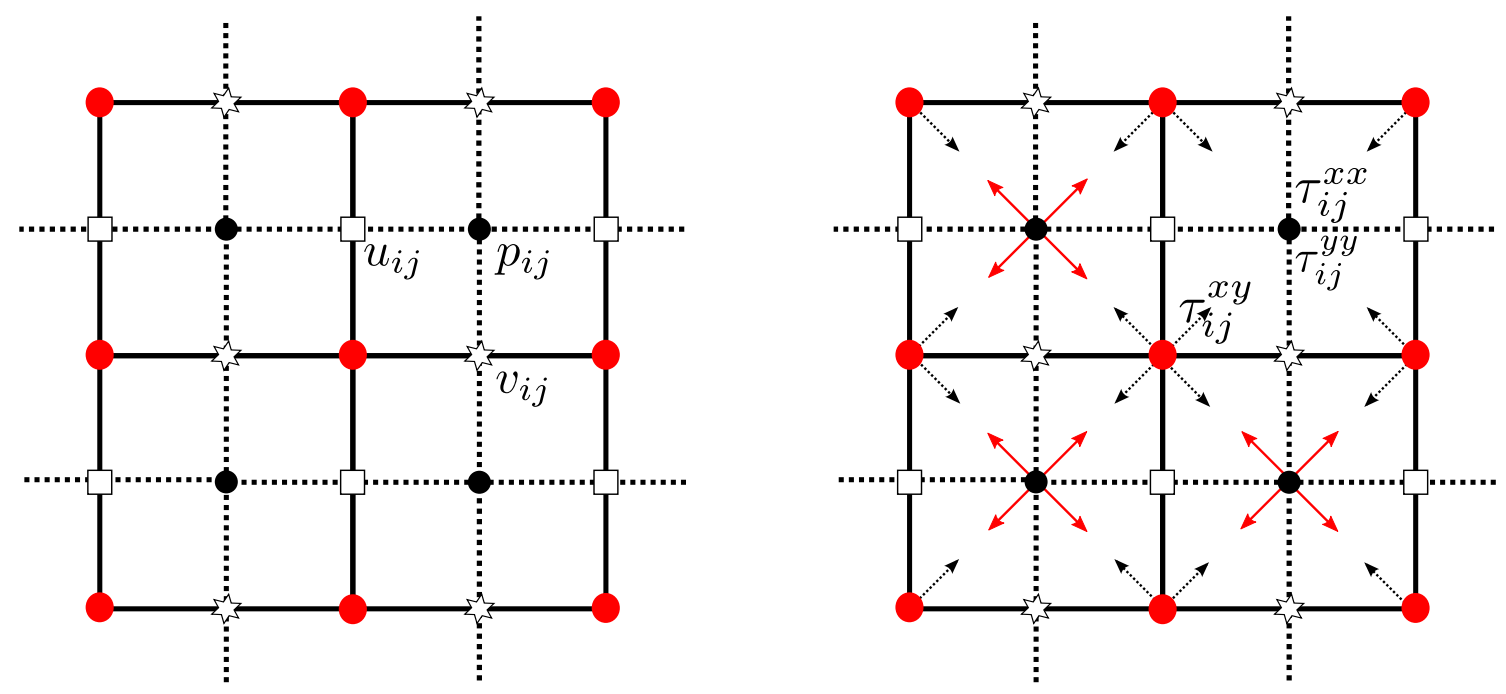

Figure 25: A typical staggered grid in the MAC approach, for velocity and pressure (left). On the right, for this same mesh, we show the locations of the various components of a symmetric tensor $\tau$ (should it be $d, \lambda$ or $D(\mathbf{u})$ of the AL algorithm): 4 points centered averages are used to recover the values of these components on the staggered locations (as materialized by the arrows).

To discretize the Stokes problem (15)-(16), we use a staggered (MAC) grid for velocity and pressure [16], as shown in figure 25 (left). The second order operator of the LHS in 
(15) is discretized with the classical associated ( $5+4$ points) MAC discretization. It is indeed shown in [21] to be consistent with AL algorithm, in the sense that its convergence does not depend on $r$. The associated scheme has a second order accuracy in space.

The boundary conditions are treated as follows (using dimensionless variables):

- Inlet and outlet: We assume that the inlet and outlet are long enough to be considered as infinitely long (see remark below). Therefore, the velocity is set equal to the velocity of the Poiseuille flow with a unit net flux, i.e.

$$
\int_{0}^{1} \tilde{u}(0, \tilde{y}) \mathrm{d} \tilde{y}=1 .
$$

We recall the velocity of the Poiseuille flow: $\tilde{\mathbf{u}}(\tilde{x}, \tilde{y})=\left(\tilde{u}_{\text {Pois }}(\tilde{y}), 0\right)$ with

$$
\tilde{u}_{\text {Pois }}(\tilde{y})=\left\{\begin{array}{lr}
\tilde{u}_{\text {plug }}-\frac{\left|\partial_{x} \tilde{P}\right|}{2}(1-\tilde{y})^{2} & \text { if } \tilde{y}_{\text {plug }} \leqslant \tilde{y} \leqslant 1 \\
\tilde{u}_{\text {plug }} & \text { if }|\tilde{y}|<\tilde{y}_{\text {plug }}=\frac{B}{\left|\partial_{x} \tilde{P}\right|} \\
\tilde{u}_{\text {plug }}-\frac{\left|\partial_{x} \tilde{P}\right|}{2}(1+\tilde{y})^{2} & \text { if }-1 \leqslant \tilde{y} \leqslant-\tilde{y}_{\text {plug }}
\end{array}\right.
$$

where $\tilde{u}_{p l u g}=\frac{\partial_{x} \tilde{P}}{2}\left(1-\tilde{y}_{p l u g}\right)^{2}$. The pressure gradient $\partial_{x} \tilde{P}$ can be computed from $B$, using the classical Buckingham equation.

- Walls: we set $\tilde{\mathbf{u}}=0$.

One issue of the numerical tests was the necessary length of the "exterior" channels (denoted as $L_{\text {inout }}$ ), in order to put a consistent boundary condition at the inlet/outlet. We do not want a too long entrance channel, for it increases the number of cells and, of course, the computation time. One way of checking the consistency is the evolution of the plug zone when this length increases. Therefore, we can assume that putting a Poiseuille flow in the inlet/outlet is consistent if the yield surface remains unchanged for a significant distance inside the domain. Figure 26 shows that for a ratio $\frac{L_{\text {inout }}}{2 D} \geqslant 1$, the yield surface remains the same in the extended entrance. Therefore, in the simulations of this paper, we set $L_{\text {inout }}=2 D$.

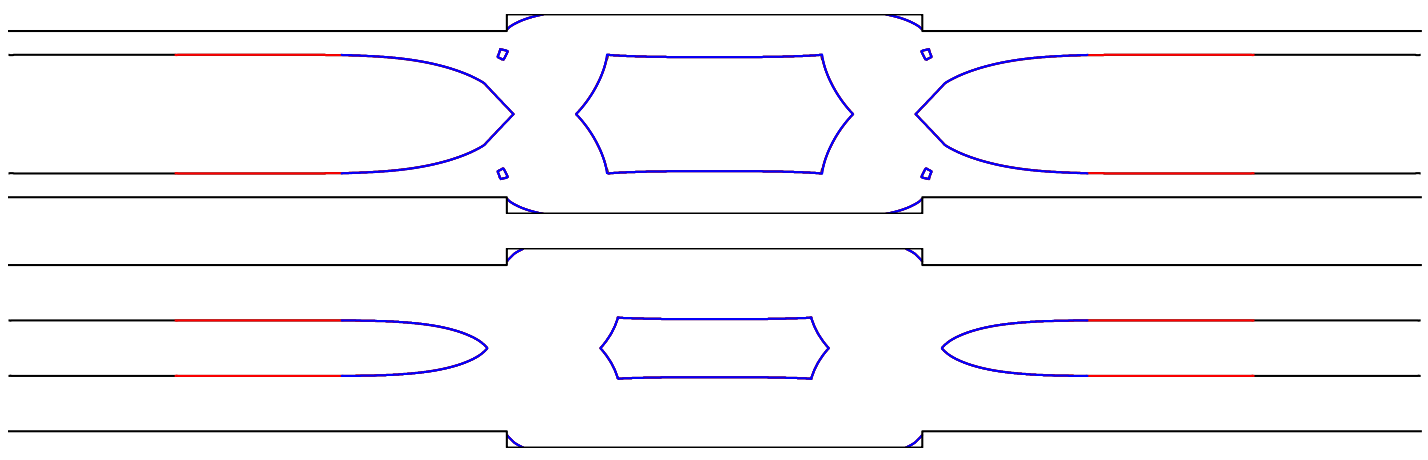

Figure 26: Superposition of the shape of the contour $|d|=10^{-10}$ for $B=20$ (top) and 2 (bottom) for 3 different aspect ratio of the entry length: $\frac{L_{\text {inout }}}{D}=6$ (black), 4 (red) and 2 (blue)

We implemented from scratch the aforementioned algorithms in a parallel (MPI) Fortran 90 code. To solve the linear system associated to (15), we embed the MUMPS library 
$[1,2]$. MUMPS is a multifrontal massively parallel sparse direct solver which performs a Gaussian factorization (so called LU decomposition method). It efficiently distributes and equilibrates the work onto the various processors and uses MPI for message passing. MUMPS is in active development and is daily used both in academia and industry for solving very large linear algebra problems.

\section{A.3 Details on the computation times}

For the typical simulations of this paper where $\Delta x=2 \cdot 10^{-2}$, a $y$-section in the cavity contains 600 points. We give in table 1 the total number of mesh points depending on the geometry.

\begin{tabular}{|l|l|l|l|l|l|l|l|}
\hline $3-3-6$ & $5-1-6$ & $5.5-.5-6$ & $3-3-12$ & $5-1-12$ & $5.5-.5-12$ & $5-1-25$ & $5-1-60$ \\
\hline $4.8 \cdot 10^{5}$ & $6.8 \cdot 10^{5}$ & $7.3 \cdot 10^{5}$ & $6.6 \cdot 10^{5}$ & $8.6 \cdot 10^{5}$ & $9.1 \cdot 10^{5}$ & $1.3 \cdot 10^{6}$ & $2.3 \cdot 10^{6}$ \\
\hline
\end{tabular}

Table 1: Number of mesh points for some $D$ - $H$ - $L$, given in this order on the first row.

For the largest $L$, which are the more demanding geometries, we made the simulations on a cluster with $n_{p}=16$ processors. In particular, for $L=25, D=5, H=1$ and $B=20$ (which is shown on figure 4), the computational cost as a function of $\Delta x$ is shown in table 2 .

\begin{tabular}{|l|l|l|l|}
\hline$\Delta x$ & $2 \cdot 10^{-2}$ & $1 \cdot 10^{-2}$ & $8 \cdot 10^{-3}$ \\
\hline Corresponding $\Delta \tilde{x}$ & $4 \cdot 10^{-3}$ & $2 \cdot 10^{-3}$ & $1.6 \cdot 10^{-3}$ \\
\hline Total number of mesh points & $1.25 \cdot 10^{6}$ & $5.0 \cdot 10^{6}$ & $7.8 \cdot 10^{6}$ \\
\hline AL iterations number & 16508 & 9425 & 9349 \\
\hline Sum of all Stokes iterations & 45162 & 34615 & 30513 \\
\hline CPU time & $5 \mathrm{~h} 30$ & $18 \mathrm{~h} 15$ & $32 \mathrm{~h} 30$ \\
\hline
\end{tabular}

Table 2: CPU times associated to the mesh refinement study of Fig. $4(D=5, H=1$, $L=25$ and $B=20), n_{p}=16$.

We notice that refining the mesh increases the total CPU time but it also helps to converge faster in the AL loop since the number of iterations is significantly reduced.

The other simulations were performed either on a personal computer with $n_{p}=1$ processor or by using the cluster with $n_{p}=16$ processors for the most demanding ones. Some examples of CPU time are given in table 3.

\begin{tabular}{|l|l|l|l|}
\hline$D-H-L$ & $3-3-6$ & $5-1-25$ & $5-1-60$ \\
\hline$n_{p}$ & 1 & 1 & 16 \\
\hline AL iterations number & 4618 & 13339 & 40000 \\
\hline Sum of all Stokes iterations & 12261 & 40587 & 70936 \\
\hline CPU time & $2 \mathrm{~h} 30$ & $22 \mathrm{~h} 45$ & $20 \mathrm{~h} 15$ \\
\hline
\end{tabular}

Table 3: CPU times for various geometries at $B=50$.

It is worth noting that even though $N_{\max , B}$ was attained for $(D, H, L)=(5,1,60)$, the residue of the $\mathrm{AL}$ is equal to $6.5 \cdot 10^{-12}$ (see figure 27 ) for $B=50$, which is already quite 
accurate.

We present in figure 27 a typical evolution of the AL residue with the associated evolution of the number of (15) linear problems solved for $(D, H, L)=(5,1,60)$ and $B=50$ (see also table 3, right). It appears that this number decreases dramatically during the first iterations of the AL loop. Very quickly the number of linear systems solved falls to 2 or 3 (or even 1 for the last iterations) per AL iteration. This means that the method of resolution of the Stokes subproblem does not cause much time loss (except for the first iterations of $\mathrm{AL}$ ) and, at the same time, ensure to get a velocity field which is divergencefree with a very good accuracy.

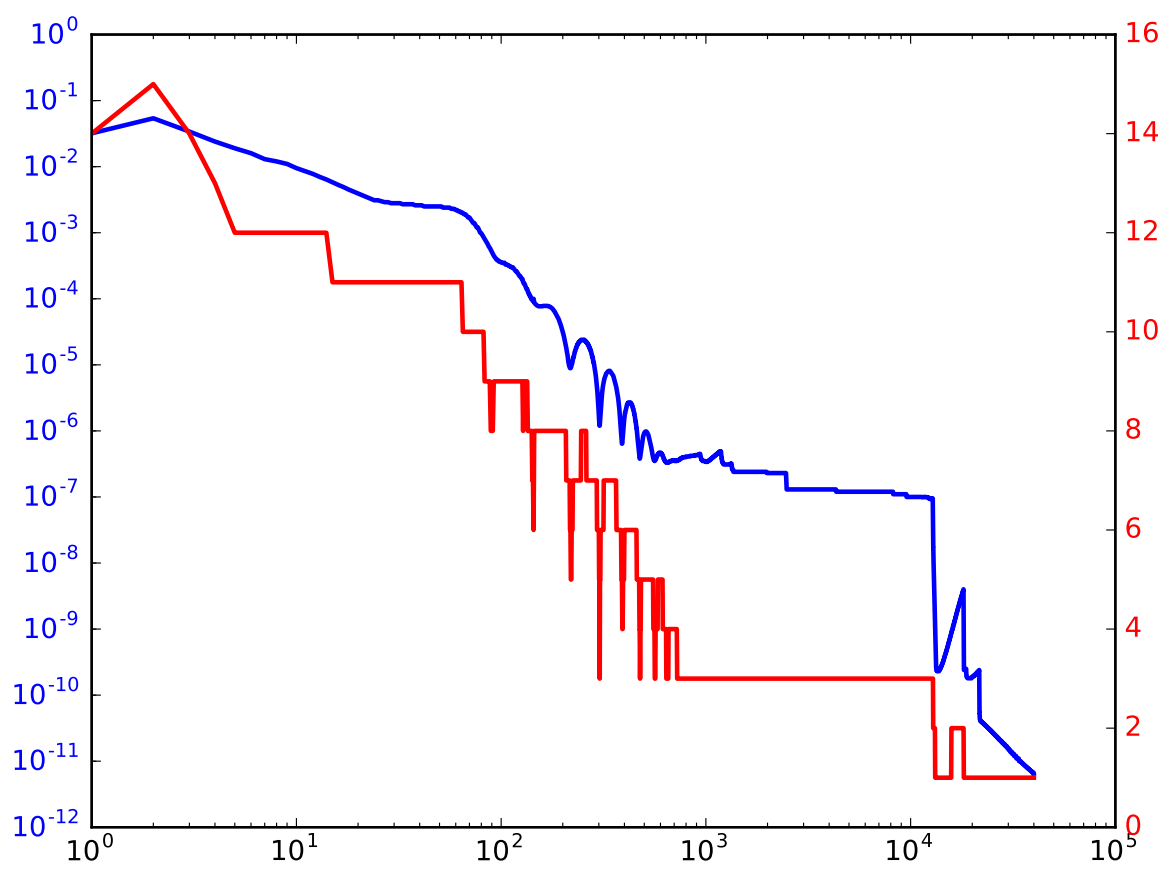

Figure 27: Convergence of the $\mathrm{AL}$ for $(D, H, L)=(5,1,60)$ and $B=50$. Values of the residue $\left\|D\left(\mathbf{u}^{i}\right)-d^{i}\right\|_{L^{2}}$ (blue curve, left ordinates) and number of (15) linear systems solved (red curve, right ordinates), as a function of the iteration number $i$ during Algorithm 1.

It is not so easy to find computation times for such kind of Bingham flows in the literature. One reference we have for such simulations is the $\mathrm{PhD}$ thesis of $\mathrm{T}$. Chevalier [5]. We reproduce a simulation for its most refined mesh which contains 19436 elements. Due to difference of the meshing method, it was not possible to put exactly the same number in our mesh, so we put 30000 elements $\left(\Delta x=8 \cdot 10^{-2}\right)$ but we used exactly the same geometry as in [5] $\left(D=H=2, L=4\right.$ and $\left.L_{\text {inout }}=8\right)$. For this test, Chevalier mentioned a CPU time of 10 hours (apparently on a single processor) while our code (also with $n_{p}=1$ ) runs between 20 and 40 minutes for various Bingham numbers. Consequently, even with more elements than in [5], CPU times of our code are at least 15 times shorter than with the code used by Chevalier.

\section{A.4 More details on $|D(\mathbf{u})|$ for longer cavities}

For completeness, in figure 28, we give the total strain rate fields for the two geometries studied in the configuration of Luu et al. [19] of section 4. This completes Fig. 8 (which actually shows the same simulations) by allowing to see the details of the pseudo-plugs. 

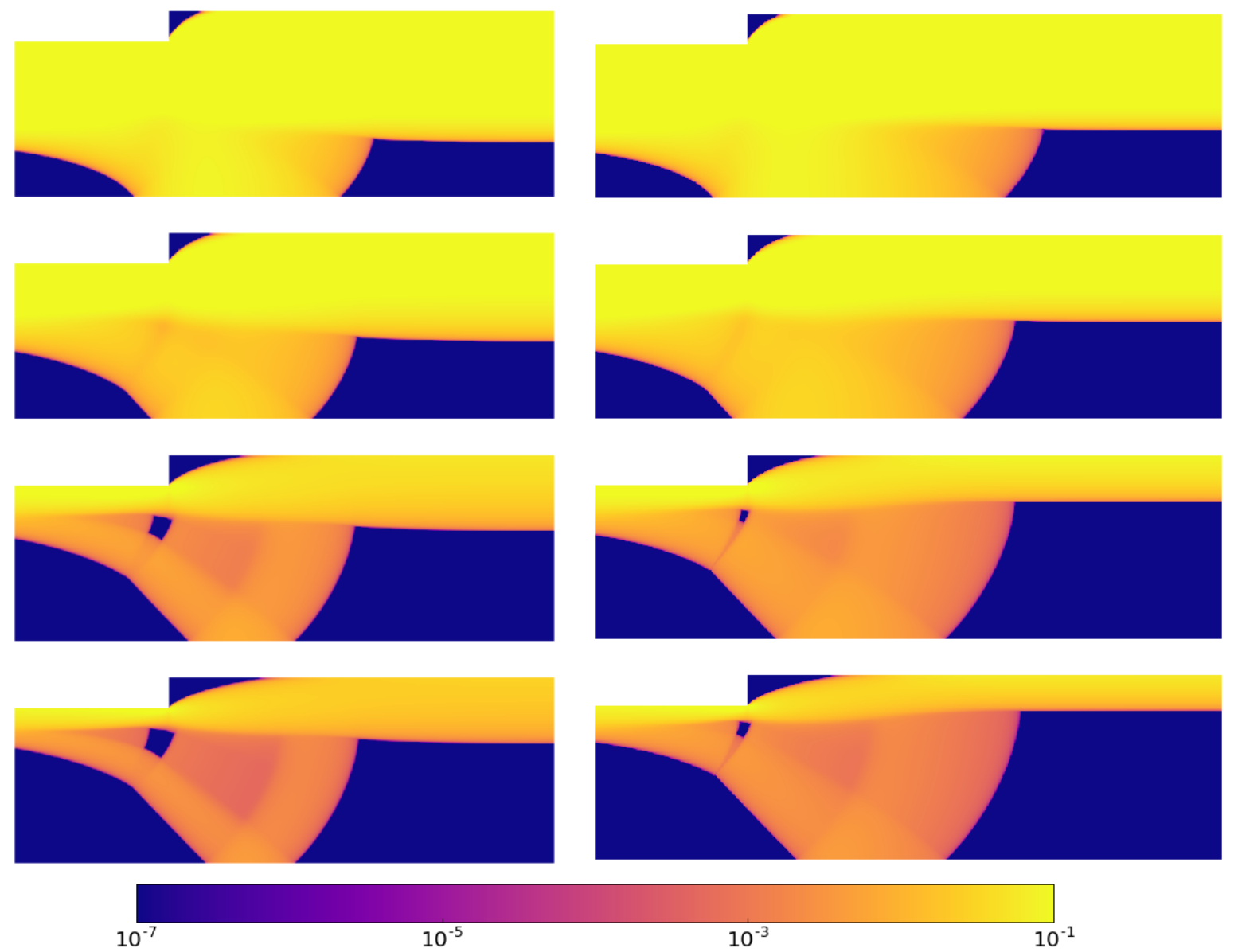

Figure 28: $|D(\mathbf{u})|$ for $H=1, D=5$ and $L=25$ (Left) or $L=60$ (Right). From top to bottom: $B=2,5,25$ and 50 .

\section{A.5 More details on pressure drops}

In figure 29, we show some examples of pressure drops for the same geometry as in figure 10. We observe that even if the material follows a frustrated regime, the pressure drop is quasi-linear in the cavity (figure 29, right). We also recover a linear pressure in the entrance and exit channels, whose slope is very close to the one imposed at the entrance and based on the Poiseuille theory in the infinite channel (see also figures 12 and 13). Of course the computed slopes in our geometry are not the same as the Poiseuille flow, due to the influence of the cavity.

Note that, following the details of section 2.2, we decide to take a pressure cut on a horizontal line which is defined at a $\tilde{y}$ which is in the middle of the fluid part of the entrance/exit channels. This choice can be discussed (for instance Roustaei et al. [29] take the averaged pressure along $\tilde{y}$ ) but our point here is to say that the pressure has only a meaning in the fluid part (and not in the rigid zone). As a consequence, we do not want to compute averaged pressure along $\tilde{y}$. To minimize the role of the plastic zone as much as possible, we were led to choose this cut along a horizontal line mentioned above. As a final remark, it can be seen that in the entrance/exit channels, the pressure is almost independent of $\tilde{y}$, as a consequence, in these zones, the pressure cut along the line and an average over $\tilde{y}$ are very close. This is another argument which leads us to the above choice. 

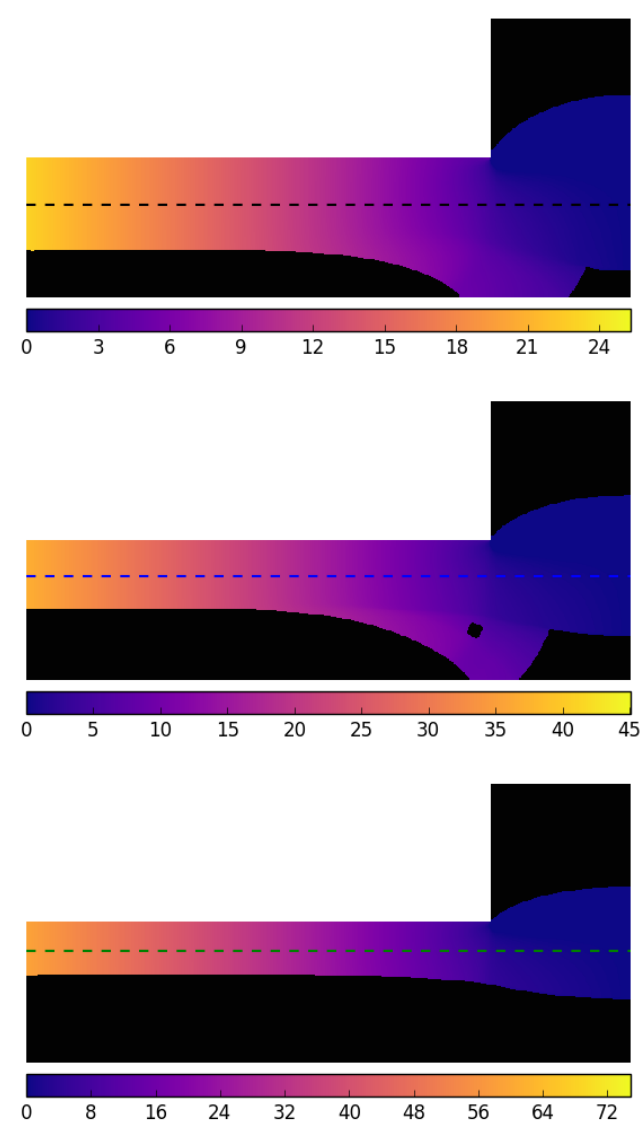

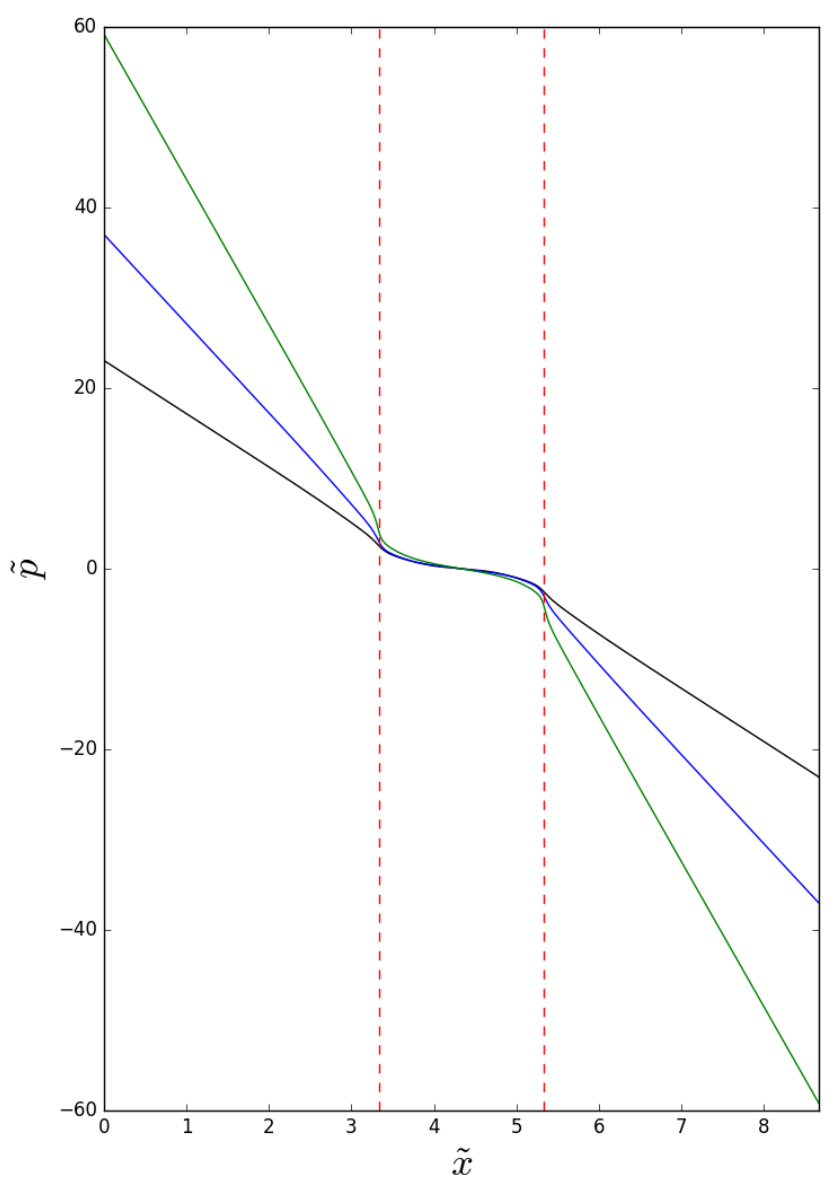

Figure 29: Pressure for $\delta=0.5, h=2$. Left: pressure fields and the dashed line, indicating the pressure cut shown on the right, for $B=2,5,10$ from top to bottom, respectively. Right: pressure drop along the $\tilde{x}$ axis for $B=2$ (black), 5 (blue), 10 (green).

\section{References}

[1] P. Amestoy, I. Duff, J. L'Excellent, and J. Koster. A Fully Asynchronous Multifrontal Solver Using Distributed Dynamic Scheduling. SIAM Journal on Matrix Analysis and Applications, 23(1):15-41, January 2001. 4, 27

[2] Patrick R. Amestoy, Abdou Guermouche, Jean-Yves L'Excellent, and Stéphane Pralet. Hybrid scheduling for the parallel solution of linear systems. Parallel Computing, 32(2):136-156, February 2006. 4, 27

[3] E. C. Bingham. Fluidity and plasticity. Mc Graw-Hill, 1922. 2

[4] Gilmer R. Burgos, Andreas N. Alexandrou, and Vladimir Entov. On the determination of yield surfaces in Herschel-Bulkley fluids. Journal of Rheology (1978-present), 43(3):463-483, May 1999. 2

[5] T. Chevalier. Ecoulements de fluides à seuil en milieux confinés (Flow of yield stress fluids in confined geometries). PhD thesis, Université Paris-Est, October 2013. 28

[6] T. Chevalier, S. Rodts, X. Chateau, J. Boujlel, M. Maillard, and P. Coussot. Boundary layer (shear-band) in frustrated viscoplastic flows. EPL (Europhysics Letters), 102(4):48002, 2013. 1, 2, 3, 10, 12, 14, 21, 22 
[7] Paulo R. de Souza Mendes, Monica F. Naccache, Priscilla R. Varges, and Flavio H. Marchesini. Flow of viscoplastic liquids through axisymmetric expansions-contractions. Journal of Non-Newtonian Fluid Mechanics, 142(1-3):207-217, March 2007. 2

[8] E. J. Dean, R. Glowinski, and G. Guidoboni. On the numerical simulation of Bingham visco-plastic flow: old and new results. Journal of Non Newtonian Fluid Mechanics, 142:36-62, 2007. 4

[9] F. Delbos, J. Ch. Gilbert, R. Glowinski, and D. Sinoquet. Constrained optimization in seismic reflection tomography: a Gauss-Newton augmented Lagrangian approach. Geophysical Journal International, 164(3):670-684, 2006. 25

[10] G. Duvaut and J.-L. Lions. Inequalities in mechanics and physics. Springer-Verlag, 1976. 2

[11] M. Fortin and R. Glowinski. Augmented Lagrangian methods: applications to the numerical solution of boundary-value problems. North-Holland, 1983. 24, 25

[12] I. A. Frigaard and C. Nouar. On the usage of viscosity regularisation methods for visco-plastic fluid flow computation. Journal of Non-Newtonian Fluid Mechanics, 127(1):1-26, April 2005. 2

[13] R. Glowinski. Numerical Methods for Fluids (Part 3), volume 9 of Handbook of Numerical Analysis. Elsevier, 2003. 24

[14] R. Glowinski and P. Le Tallec. Augmented Lagrangian and operator-splitting methods in nonlinear mechanics, volume 9 of SIAM Studies in Applied Mathematics. Society for Industrial and Applied Mathematics (SIAM), 1989. 4

[15] R. Glowinski and A. Wachs. On the numerical simulation of viscoplastic fluid flow. In R. Glowinski and J. Xu, editors, Numerical Methods for Non-Newtonian Fluids, volume 16 of Handbook of Numerical Analysis, pages 483 - 717. Elsevier, 2011. 2, 3, 24,25

[16] Francis H. Harlow and J. Eddie Welch. Numerical calculation of time-dependent viscous incompressible flow of fluid with free surface. Physics of Fluids, 8(12):21822189, 1965. 25

[17] K. Hohenemser and W. Prager. Über die Ansätze der Mechanik isotroper Kontinua. ZAMM - Journal of Applied Mathematics and Mechanics / Zeitschrift für Angewandte Mathematik und Mechanik, 12(4):216-226, January 1932. 2

[18] A. A. Il'iushin. Deformation of a viscous-plastic plastic body (in Russian). Uch. zap. MGU, Mekhanika, 1940. 2

[19] Li-Hua Luu, Pierre Philippe, and Guillaume Chambon. Experimental study of the solid-liquid interface in a yield-stress fluid flow upstream of a step. Physical Review E, 91(1):013013, January 2015. 1, 2, 3, 4, 7, 16, 17, 18, 19, 20, 22, 28

[20] P.P. Mosolov and V.P. Miasnikov. Variational methods in the theory of the fluidity of a viscous-plastic medium (translated from PMM, Prikladnaya Matematika i Mekhanika). Journal of Applied Mathematics and Mechanics, 29(3):545 - 577, 1965. 
[21] E. A. Muravleva and M. A. Olshanskii. Two finite-difference schemes for calculation of Bingham fluid flows in a cavity. Russian Journal of Numerical Analysis and Mathematical Modelling, 23(6):615-634, 2008. 3, 25, 26

[22] Larisa Muravleva. Uzawa-like methods for numerical modeling of unsteady viscoplastic Bingham medium flows. Applied Numerical Mathematics, 93:140-149, July 2015. 3

[23] J. G. Oldroyd. Two-dimensional plastic flow of a Bingham solid, a plastic boundary layer theory for slow motion. Mathematical Proceedings of the Cambridge Philosophical Society, 43(3):383-395, 1947. 12

[24] William Prager. On slow visco-plastic flow. Technical report 84, Graduate Division of Applied Mathematics. Brown University., Providence, R.I., December 1952. 2

[25] A. Putz, I. A. Frigaard, and D. M. Martinez. On the lubrication paradox and the use of regularisation methods for lubrication flows. Journal of Non-Newtonian Fluid Mechanics, 163(1-3):62-77, November 2009. 5

[26] Nicolas Roquet and Pierre Saramito. An adaptive finite element method for Bingham fluid flows around a cylinder. Computer Methods in Applied Mechanics and Engineering, 192(31-32):3317-3341, August 2003. 2

[27] A. Roustaei. Yield stress fluid flows in uneven geometries: applications to the oil and gas industry. PhD thesis, University of British Columbia (Vancouver), May 2016. 5

[28] A. Roustaei and I. A. Frigaard. The occurrence of fouling layers in the flow of a yield stress fluid along a wavy-walled channel. Journal of Non-Newtonian Fluid Mechanics, 198:109-124, August 2013. 2, 4, 10

[29] A. Roustaei, A. Gosselin, and I. A. Frigaard. Residual drilling mud during conditioning of uneven boreholes in primary cementing. Part 1: Rheology and geometry effects in non-inertial flows. Journal of Non-Newtonian Fluid Mechanics, 220:87-98, June 2015. 2, 4, 5, 7, 29

[30] Pierre Saramito. Efficient $C++$ finite element computing with Rheolef. CNRS-CCSD ed., 2015. http://cel.archives-ouvertes.fr/cel-00573970. 2

[31] Theodore Schwedoff. Recherches expérimentales sur la cohésion des liquides. J. Phys. Theor. Appl., 8(1):341 - 359, 1889. 2

[32] Guillaume Vinay, Anthony Wachs, and Jean-François Agassant. Numerical simulation of non-isothermal viscoplastic waxy crude oil flows. Journal of Non-Newtonian Fluid Mechanics, 128(2-3):144-162, July 2005. 3, 25 